\title{
Are classes of deterministic integrands for fractional Brownian motion on an interval complete?
}

\author{
VLADAS PIPIRAS* and MURAD S. TAQQU** \\ Department of Mathematics, Boston University, 111 Cummington St., Boston MA 02215, USA. \\ E-mail:*pipiras@math.bu.edu; **murad@math.bu.edu
}

Let $B_{H}$ be a fractional Brownian motion with self-similarity parameter $H \in(0,1)$ and $a>0$ be a fixed real number. Consider the integral $\int_{0}^{a} f(u) \mathrm{d} B_{H}(u)$, where $f$ belongs to a class of non-random integrands $\Lambda_{H, a}$. The integral will then be defined in the $L^{2}(\Omega)$ sense. One would like $\Lambda_{H, a}$ to be a complete inner-product space. This corresponds to a desirable situation because then there is an isometry between $\Lambda_{H, a}$ and the closure of the span generated by $B_{H}(u), 0 \leqslant u \leqslant a$. We show in this work that, when $H \in\left(\frac{1}{2}, 1\right)$, the classes of integrands $\Lambda_{H, a}$ one usually considers are not complete inner-product spaces even though they are often assumed in the literature to be complete. Thus, they are isometric not to $\overline{\operatorname{sp}}\left\{B_{H}(u), 0 \leqslant u \leqslant a\right\}$ but only to a proper subspace. Consequently, there are (random) elements in that closure which cannot be represented by functions $f$ in $\Lambda_{H, a}$. We also show, in contrast to the case $H \in\left(\frac{1}{2}, 1\right)$ that there is a class of integrands for fractional Brownian motion $B_{H}$ with $H \in\left(0, \frac{1}{2}\right)$ on an interval $[0, a]$ which is a complete inner-product space.

Keywords: completeness; fractional Brownian motion; fractional integrals and derivatives; innerproduct spaces; integration in the $L^{2}$ sense

\section{Introduction}

A fractional Brownian motion (FBM) $B_{H}=\left\{B_{H}(u)\right\}_{u \in \mathbb{R}}$ with self-similarity parameter $H \in(0,1)$ is a zero-mean Gaussian process which has stationary increments and is selfsimilar with index $H$, that is, for any $c>0$,

$$
\left\{B_{H}(c u)\right\}_{u \in \mathbb{R}} \stackrel{d}{=}\left\{c^{H} B_{H}(u)\right\}_{u \in \mathbb{R}},
$$

where $\stackrel{d}{=}$ stands for equality in the sense of finite-dimensional distributions. For notational simplicity we will use another parametrization of FBM. Let

$$
\kappa=H-\frac{1}{2}
$$

so that the range $H \in(0,1)$ now corresponds to the range $\kappa \in\left(-\frac{1}{2}, \frac{1}{2}\right)$. We will denote the

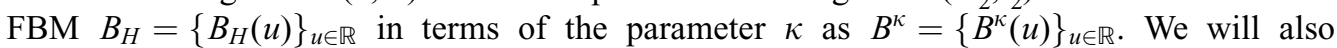
assume that the FBM $B^{\kappa}$ is standard, i.e. $\mathrm{E}\left(B^{\kappa}(1)\right)^{2}=1$. By using stationarity of the 
increments and the self-similarity with index $\kappa+\frac{1}{2}$ of a standard FBM $B^{\kappa}$, it is easy to see that the covariance function of $B^{\kappa}$ is given by

$$
\Gamma^{\kappa}(u, v)=\mathrm{E} B^{\kappa}(u) B^{\kappa}(v)=\frac{1}{2}\left\{|u|^{2 \kappa+1}+|v|^{2 \kappa+1}-|u-v|^{2 \kappa+1}\right\}, \quad u, v \in \mathbb{R} .
$$

When $\kappa=0$, the FBM $B^{\kappa}=B^{0}$ is the usual Brownian motion which has independent increments. If $\kappa \neq 0$, the increments of the $\mathrm{FBM} B^{\kappa}$ are no longer independent: they are positively correlated if $\kappa \in\left(0, \frac{1}{2}\right)$, and negatively correlated if $\kappa \in\left(-\frac{1}{2}, 0\right)$. Moreover, when $\kappa \in\left(0, \frac{1}{2}\right)$, the dependence at large time lags is so strong that the series $\sum_{k=1}^{\infty} \Gamma^{\kappa}(1, k)$ diverges. In this case, one says that the FBM $B^{\kappa}$ exhibits long-range dependence. (For more information on FBM see, for example, Samorodnitsky and Taqqu 1994).

In this work we deal with questions related to the $L^{2}(\Omega)$-integration of deterministic functions with respect to the FBM $B^{\kappa}$ when $\kappa \in\left(-\frac{1}{2}, \frac{1}{2}\right)$. To define such integrals, one typically starts with an inner-product space $\left(\mathcal{C},(\cdot, \cdot)_{c}\right)$ of functions on a region of integration $R$ (say, $R=\mathbb{R}$ or $[0, a]$ with $a>0$ ) such that $\left(1_{[0, s)}, 1_{[0, t)}\right) c=\mathrm{E} B^{\kappa}(s) B^{\kappa}(t)$ for all $s, t \in R$. Let $\overline{\mathrm{sp}}_{R}\left(B^{\kappa}\right)$ be the closure in $L^{2}(\Omega)$ of all posible linear combinations of the increments of FBM on $R$. If the map $1_{[0, t)} \mapsto B^{\kappa}(t)$ extends to the isometry between this class of functions $\mathscr{C}$ and the space $\overline{\mathrm{sp}}_{R}\left(B^{\kappa}\right)$, then the resulting isometry map is called the integral in the $L^{2}(\Omega)$ sense with respect to FBM of functions from $\mathcal{C}$.

The extension step is usually taken for granted (i.e. not proved). So, for example, when $\kappa \in\left(0, \frac{1}{2}\right)$ and the region of integration $R$ is $[0, a]$ with $a>0$, Carmona et al. (1999) and Kleptsyna et al. (1999b) defined the class of integrands for FBM

$$
\Lambda_{a}^{\kappa}=\left\{f:[0, a] \mapsto \mathbb{R} \text { such that } \int_{0}^{a}\left[s^{-\kappa}\left(I_{a-}^{\kappa} u^{\kappa} f(u)\right)(s)\right]^{2} \mathrm{~d} s<\infty\right\},
$$

where $I_{a-}^{\kappa}$ is a fractional integral operator defined in Section 2 below (see (2.1)). Another class of integrands, considered by Duncan et al. (2000), Kleptsyna et al. (1999a) and Norros et al. (1999), is given by

$$
|\Lambda|_{a}^{\kappa}=\left\{f:[0, a] \mapsto \mathbb{R} \text { such that } \int_{0}^{a} \int_{0}^{a}|f(u)\|f(v)\| u-v|^{2 \kappa-1} \mathrm{~d} u \mathrm{~d} v<\infty\right\} .
$$

The classes of integrands $\Lambda_{a}^{\kappa}$ and $|\Lambda|_{a}^{\kappa}$ are assumed to be inner-product spaces with the inner products

$$
(f, g)_{\Lambda_{a}^{\kappa}}=\frac{\pi \kappa(2 \kappa+1)}{\Gamma(1-2 \kappa) \sin \pi \kappa} \int_{0}^{a} s^{-2 \kappa}\left(I_{a-}^{\kappa} u^{\kappa} f(u)\right)(s)\left(I_{a-}^{\kappa} u^{\kappa} g(u)\right)(s) \mathrm{d} s
$$

$\left(\Gamma(p)=\int_{0}^{\infty} \mathrm{e}^{-v} v^{p-1} \mathrm{~d} v, p>0\right.$, is the gamma function) and

$$
(f, g)_{|\Lambda|_{a}^{\kappa}}=\kappa(2 \kappa+1) \int_{0}^{a} \int_{0}^{a} f(u) g(v)|u-v|^{2 \kappa-1} \mathrm{~d} u \mathrm{~d} v,
$$

respectively. (For details on the construction of classes of integrands $\Lambda_{a}^{\kappa}$ and $|\Lambda|_{a}^{\kappa}$, see Section 4 below.) All the authors claimed that both $\Lambda_{a}^{\kappa}$ and $|\Lambda|_{a}^{\kappa}$ are isometric to the Gaussian space $\overline{\mathrm{sp}}_{[0, a]}\left(B^{\kappa}\right)$. Since $\overline{\mathrm{sp}}_{[0, a]}\left(B^{\kappa}\right)$ is a complete inner-product space, both $\Lambda_{a}^{\kappa}$ and $|\Lambda|_{a}^{\kappa}$ necessarily have to be complete inner-product spaces as well. We will show in this work that, when $\kappa \in\left(0, \frac{1}{2}\right)$, neither the space of functions $\Lambda_{a}^{\kappa}$ nor the space of functions $|\Lambda|_{a}^{\kappa}$ is a complete 
inner-product space. Thus, they cannot be isometric to $\overline{\operatorname{sp}}_{[0, a]}\left(B^{\kappa}\right)$ itself: in fact, they are isometric only to proper linear subspaces of $\overline{\operatorname{sp}}_{[0, a]}\left(B^{\kappa}\right)$. Consequently, there are (random) elements in $\overline{\mathrm{sp}}_{[0, a]}\left(B^{\kappa}\right)$ which cannot be represented by functions $f$ belonging to either $\Lambda_{a}^{\kappa}$ or $|\Lambda|_{a}^{\kappa}$.

One area of applications of integrals with respect to FBM, where completeness of classes of integrands is relevant, involves prediction problems. Consider, for example, the problem of predicting the value of an FBM at some future time $t>0$ given its past from time 0 to time $a$ (with $a<t$ ), or in mathematical terms, of computing the conditional expectation $X=\mathrm{E}\left(B^{\kappa}(t) \mid B^{\kappa}(s), s \in[0, a]\right)$. It is well known that $X \in \overline{\mathrm{sp}}_{[0, a]}\left(B^{\kappa}\right)$. One would expect that $X=\int_{0}^{a} f \mathrm{~d} B^{\kappa}$. But, when $\kappa>0$, in view of the above-mentioned incompleteness results, there may be no $f$ belonging to $\Lambda_{a}^{\kappa}$ or $|\Lambda|_{a}^{\kappa}$ such that $X=\int_{0}^{a} f \mathrm{~d} B^{\kappa}$. In fact, such an $f$ exists as is shown in Section 7 below, where the prediction problem for FBM is discussed.

Although $a>0$ is assumed to be a real number, our results are also valid in the case $a=\infty$. (The space of integrands $|\Lambda|_{a}^{\kappa}$ with $a=\infty$ is considered in Duncan et al. 2000 and Norros et al. 1999). As shown in Section 6 below, when $\kappa \in\left(0, \frac{1}{2}\right)$ incompleteness of classes of integrands in the case $a=\infty$ can in principle be deduced from that of classes of integrands in the case $a>0$.

The results described above are in the same spirit as those of Pipiras and Taqqu (2000), where the integration is over $\mathbb{R}$. The $\mathbb{R}$ set-up, however, is quite different from that of $[0, a]$ considered here. When $\kappa \in\left(0, \frac{1}{2}\right)$, an $\mathbb{R}$-analogue of the space of integrands $\Lambda_{a}^{\kappa}$ is the innerproduct space

$$
\Lambda^{\kappa}=\left\{f: \mathbb{R} \mapsto \mathbb{R} \text { such that } \int_{\mathbb{R}}\left[\left(I_{-}^{\kappa} f\right)(s)\right]^{2} \mathrm{~d} s<\infty\right\}
$$

with the inner product

$$
(f, g)_{\Lambda^{\kappa}}=\frac{\Gamma(\kappa+1)^{2}}{c_{1}(\kappa)^{2}} \int_{\mathbb{R}}\left(I_{-}^{\kappa} f\right)(s)\left(I_{-}^{\kappa} g\right)(s) \mathrm{d} s
$$

where $\left(I_{-}^{\kappa} f\right)(s)=(\Gamma(\kappa))^{-1} \int_{\mathbb{R}} f(u)(u-s)_{+}^{\kappa-1} \mathrm{~d} u, s \in \mathbb{R}$, is a fractional integral on $\mathbb{R}$ and $c_{1}(\kappa)$ is some constant which depends on $\kappa$. As shown in Theorem 3.2 of Pipiras and Taqqu (2000), the inner-product space $\Lambda^{\kappa}$ is not complete. We do not know whether one can use this to conclude immediately that $\Lambda_{a}^{\kappa}$ is incomplete. We shall provide a proof that relies indirectly on the incompleteness of $\Lambda^{\kappa}$. Obtaining results concerning integration over $[0, a]$ or $[0, \infty)$ is important in practice (most of the papers quoted above consider integration over this range).

Suppose now that the parameter $\kappa$ belongs to the range $\left(-\frac{1}{2}, 0\right)$. (This range corresponds to $H \in\left(0, \frac{1}{2}\right)$ or no long-range dependence.) It is natural to ask whether there is a class of integrands for the FBM $B^{\kappa}$ with $\kappa \in\left(-\frac{1}{2}, 0\right)$ on an interval $[0, a]$ which is a complete innerproduct space. It turns out that such a class of integrands exists. It is an inner-product space

$$
\Lambda_{a}^{\kappa}=\left\{f: \exists \phi_{f} \in L^{2}[0, a] \text { such that } f(u)=u^{-\kappa}\left(I_{a-}^{-\kappa} s^{\kappa} \phi_{f}(s)\right)(u)\right\},
$$

with the inner product 


$$
\begin{aligned}
(f, g)_{\Lambda_{a}^{\kappa}} & =\frac{\pi \kappa(2 \kappa+1)}{\Gamma(1-2 \kappa) \sin \pi \kappa} \int_{0}^{a} s^{-2 \kappa}\left(I_{a-}^{\kappa} u^{\kappa} f(u)\right)(s)\left(I_{a-}^{\kappa} u^{\kappa} g(u)\right)(s) \mathrm{d} s \\
& =\frac{\pi \kappa(2 \kappa+1)}{\Gamma(1-2 \kappa) \sin \pi \kappa} \int_{0}^{a} \phi_{f}(s) \phi_{g}(s) \mathrm{d} s,
\end{aligned}
$$

where $\phi_{f}, \phi_{g} \in L^{2}[0, a]$ are associated with the functions $f$ and $g$, respectively, by definition (1.8), and $I_{a-}^{\kappa}$ with $\kappa \in\left(-\frac{1}{2}, 0\right)$ are fractional derivatives of order $-\kappa$. (The fractional derivatives $I_{a-}^{\kappa}$ with $\kappa \in\left(-\frac{1}{2}, 0\right)$ are defined in Section 2 below. They satisfy the property $I_{a-}^{\kappa} I_{a-}^{-\kappa} \phi=\phi$ for any $\phi \in L^{1}[0, a]$ and $\kappa \in\left(-\frac{1}{2}, 0\right)$, which explains the equality of (1.9) and (1.10).) For the construction of the class of integrands $\Lambda_{a}^{\kappa}$ with $\kappa \in\left(-\frac{1}{2}, 0\right)$ and for the proof of its completeness, see Section 4 below.

This work is organized as follows. In Section 2 we provide a quick review of fractional integrals and derivatives that are used in this work. Then, in Section 3, we represent FBM on an interval in terms of these fractional integrals and derivatives. We use this representation in Section 4 to construct the classes of integrands $\Lambda_{a}^{\kappa}$ with $\kappa \in\left(-\frac{1}{2}, \frac{1}{2}\right)$ and $|\Lambda|_{a}^{\kappa}$ with $\kappa \in\left(0, \frac{1}{2}\right)$. We also show, in Section 4 , that $\Lambda_{a}^{\kappa}$ is a complete inner-product space when $\kappa \in\left(-\frac{1}{2}, 0\right)$ and, in Section 5 , that $\Lambda_{a}^{\kappa}$ and $|\Lambda|_{a}^{\kappa}$ are not complete inner-product spaces when $\kappa \in\left(0, \frac{1}{2}\right)$. In section 6 we deal with the case $a=\infty$. We consider the prediction problem for FBM in Section 7. Finally, in Section 8 we prove some of the results of Section 4.

\section{Fractional integrals and derivatives}

An exhaustive source on fractional integrals and derivatives is the book by Samko et al. (1993). For the reader's convenience, we provide below definitions of those fractional operators that are used throughout this paper and also list a number of their properties.

Consider the interval $[0, a]$ and let $s \in[0, a]$. An integral over $[0, s]$ is called left-sided and one over $[s, a]$ is called right-sided. The right-sided fractional integral of order $\alpha>0$ on an interval $[0, a]$ of a function $f \in L^{1}[0, a]$ is defined by

$$
\left(I_{a-}^{\alpha} f\right)(s)=\frac{1}{\Gamma(\alpha)} \int_{0}^{a} f(u)(u-s)_{+}^{\alpha-1} \mathrm{~d} u=\frac{1}{\Gamma(\alpha)} \int_{s}^{a} f(u)(u-s)^{\alpha-1} \mathrm{~d} u, \quad s \in(0, a)
$$

(see Samko et al. 1993, p. 33). The right-sided fractional derivative of order $0<\alpha<1$ on an interval $[0, a]$ of a function $\phi$ is defined by

$$
\left(\mathcal{D}_{a-}^{\alpha} \phi\right)(u)=-\frac{1}{\Gamma(1-\alpha)} \frac{\mathrm{d}}{\mathrm{d} u} \int_{0}^{a} \phi(s)(s-u)_{+}^{-\alpha} \mathrm{d} s, \quad u \in(0, a)
$$

(see Samko et al. 1993, p. 35). If $0<\alpha<1$ and

$$
\phi(s)=\left(I_{a-}^{\alpha} f\right)(s), \quad s \in(0, a),
$$

then

$$
f(u)=\left(\mathcal{D}_{a-}^{\alpha} \phi\right)(u), \quad u \in(0, a)
$$

(see Samko et al. 1993, pp. 29-31). Hence, $\mathcal{D}_{a-}^{\alpha}$ can be viewed as an inverse of $I_{a-}^{\alpha}$. 
(Heuristically, performing the differentiation over $u$ in (2.2) yields (2.1) with $\mathcal{D}_{a-}^{\alpha}=I_{a-}^{-\alpha}$.) For this reason, we will often denote the fractional derivative $\mathcal{D}_{a-}^{\alpha}$ with $\alpha \in(0,1)$ by $I_{a-}^{-\alpha}$ and also use the notation $I_{a-}^{0}$ for the identity operator, that is, $I_{a-}^{0-} f=f$. One can also show that, for any $f \in L^{1}[0, a]$,

$$
\left(\mathcal{D}_{a-}^{\alpha} I_{a-}^{\alpha} f\right)(s)=f(s), \quad s \in(0, a)
$$

(see Samko et al. 1993, p. 24).

\section{Representation of fractional Brownian motion on an interval}

The following proposition relates FBM and the fractional integral and derivative operators on an interval introduced in Section 2. It will be used in Section 4 to construct classes of integrands for FBM on an interval $[0, a]$.

Proposition 3.1. Let $a>0$ and $B^{\kappa}$ be a standard FBM with parameter $\kappa \in\left(-\frac{1}{2}, \frac{1}{2}\right)$. Then

$$
\left\{B^{\kappa}(t)\right\}_{t \in[0, a]} \stackrel{d}{=}\left\{\sigma_{1}(\kappa) \int_{0}^{a} s^{-\kappa}\left(I_{a-}^{\kappa} u^{\kappa} 1_{[0, t)}(u)\right)(s) \mathrm{d} B^{0}(s)\right\}_{t \in[0, a]},
$$

where

$$
\sigma_{1}(\kappa)^{2}=\frac{\Gamma(\kappa)^{2} \kappa(2 \kappa+1)}{\mathrm{B}(\kappa, 1-2 \kappa)}=\frac{\pi \kappa(2 \kappa+1)}{\Gamma(1-2 \kappa) \sin \pi \kappa}
$$

and $\mathrm{B}(p, q)=\int_{0}^{1}(1-v)^{p-1} v^{q-1} \mathrm{~d} v=\Gamma(p) \Gamma(q) / \Gamma(p+q), p, q>0$, is the beta function.

Proof. Suppose first that $\kappa \in\left(0, \frac{1}{2}\right)$. It follows from $(1.1)$ that $\mathrm{d}^{2} \Gamma^{\kappa}(u, v)=$ $\kappa(2 \kappa+1)|u-v|^{2 \kappa-1} \mathrm{~d} u \mathrm{~d} v$ and hence that, for any $t_{1}, t_{2} \in \mathbb{R}$,

$$
\Gamma^{\kappa}\left(t_{1}, t_{2}\right)=\kappa(2 \kappa+1) \int_{\mathbb{R}} \int_{\mathbb{R}} 1_{\left[0, t_{1}\right)}(u) 1_{\left[0, t_{2}\right)}(u)|u-v|^{2 \kappa-1} \mathrm{~d} u \mathrm{~d} v .
$$

By making the change of variables $s=(u v) z /(\min (u, v) z+|u-v|)$ below, one can show that, for $\kappa \in\left(0, \frac{1}{2}\right)$ and $u, v \in[0, a]$,

$$
|u-v|^{2 \kappa-1}=\frac{(u v)^{\kappa}}{\mathrm{B}(\kappa, 1-2 \kappa)} \int_{0}^{a} s^{-2 \kappa}(v-s)_{+}^{\kappa-1}(u-s)_{+}^{\kappa-1} \mathrm{~d} s .
$$

Then, for $t_{1}, t_{2} \in[0, a]$, we obtain from (3.3), (3.4) and definition (2.1) that

$$
\Gamma^{\kappa}\left(t_{1}, t_{2}\right)=\frac{\Gamma(\kappa)^{2} \kappa(2 \kappa+1)}{\mathrm{B}(\kappa, 1-2 \kappa)} \int_{0}^{a}\left(s^{-\kappa}\left(I_{a-}^{\kappa} u^{\kappa} 1_{\left[0, t_{1}\right)}(u)\right)(s)\right)\left(s^{-\kappa}\left(I_{a-}^{\kappa} u^{\kappa} 1_{\left[0, t_{2}\right)}(u)\right)(s)\right) \mathrm{d} s .
$$

Hence, the process on the right-hand side of (3.1) has the same covariance structure as a standard FBM. Since it is also Gaussian and has zero mean, it is a standard FBM.

The case $\kappa \in\left(-\frac{1}{2}, 0\right)$ is more delicate. Observe first that, for $\kappa \in\left(-\frac{1}{2}, \frac{1}{2}\right)$, 


$$
\begin{aligned}
-\Gamma(1+\kappa) s^{-\kappa}\left(I_{a-}^{\kappa} u^{\kappa} 1_{[0, t)}(u)\right)(s) & =s^{-\kappa} \frac{\mathrm{d}}{\mathrm{d} s} \int_{0}^{t} u^{\kappa}(u-s)_{+}^{\kappa} \mathrm{d} u \\
& =\kappa s^{-\kappa} \int_{s}^{t} u^{\kappa-1}(u-s)_{+}^{\kappa} \mathrm{d} u-\left(\frac{t}{s}\right)^{\kappa}(t-s)_{+}^{\kappa} .
\end{aligned}
$$

Indeed, by changing, in the relation below, the order of integration and performing integration by parts, we obtain that, for any $v \in[0, a]$,

$$
\begin{aligned}
\kappa \int_{v}^{t} \int_{s}^{t} u^{\kappa-1}(u-s)_{+}^{\kappa} \mathrm{d} u \mathrm{~d} s-\int_{v}^{t} t^{\kappa}(t-s)_{+}^{\kappa} \mathrm{d} s & =\kappa \int_{v}^{t} \mathrm{~d} u u^{\kappa-1} \int_{v}^{u}(u-s)^{\kappa} \mathrm{d} s-\frac{t^{\kappa}(t-v)^{\kappa+1}}{\kappa+1} \\
& =\frac{\kappa}{\kappa+1} \int_{v}^{t} u^{\kappa-1}(u-v)^{\kappa+1} \mathrm{~d} u-\frac{t^{\kappa}(t-v)^{\kappa+1}}{\kappa+1} \\
& =-\int_{v}^{t} u^{\kappa}(u-v)_{+}^{\kappa} \mathrm{d} u .
\end{aligned}
$$

The equality of (3.5) and (3.6) then follows by taking the derivative of both sides in (3.7).

The idea now is to view (3.5) or (3.6) as the definition of the left-hand side of (3.5) for $\kappa$ complex, $|\kappa|<\frac{1}{2}$. Let $t_{1}, t_{2} \in[0, a]$ be fixed and $\kappa \in \mathbb{C}$ be such that $|\kappa|<\frac{1}{2}$. Then one can verify (see Appendix) that the function

$$
f(\kappa)=\sigma_{1}(\kappa)^{2} \int_{0}^{a} s^{-2 \kappa}\left(I_{a-}^{\kappa} u^{\kappa} 1_{\left[0, t_{1}\right)}(u)\right)(s)\left(I_{a-}^{\kappa} u^{\kappa} 1_{\left[0, t_{2}\right)}(u)\right)(s) \mathrm{d} s
$$

is analytic on $\left\{\kappa:|\kappa|<\frac{1}{2}\right\}$. On the other hand, the function

$$
g(\kappa)=\Gamma^{\kappa}\left(t_{1}, t_{2}\right)=\frac{1}{2}\left\{\left|t_{1}\right|^{2 \kappa+1}+\left|t_{2}\right|^{2 \kappa+1}-\left|t_{2}-t_{1}\right|^{2 \kappa+1}\right\}
$$

is also analytic on $\left\{\kappa:|\kappa|<\frac{1}{2}\right\}$. Since the analytic functions $f$ and $g$ coincide for real $\kappa \in\left(0, \frac{1}{2}\right)$, standard results of complex analysis (see, for example, Conway 1995, p. 79) imply that they have to coincide on $\left\{\kappa:|\kappa|<\frac{1}{2}\right\}$. In particular, $f(\kappa)=g(\kappa)$ for real $\kappa \in\left(-\frac{1}{2}, 0\right)$. But this means that the Gaussian zero-mean process on the right-hand side of (3.1) has the same covariance structure as a standard FBM for $\kappa \in\left(-\frac{1}{2}, 0\right)$ as well. Hence, it is a standard FBM.

Remark 3.1. The representation of FBM with the kernel function on the right-hand side of (3.5) is also given in Kleptsyna et al. (1999b). The representation with the kernel function in (3.6) can be found in Norros et al. (1999). Using again an analyticity argument, one can also show that, for $\kappa \in\left(-\frac{1}{2}, \frac{1}{2}\right)$ and $s, t \in[0, a]$,

$$
\Gamma(\kappa+1) s^{-\kappa}\left(I_{a-}^{\kappa} u^{\kappa} 1_{[0, t)}(u)\right)(s)=(t-s)_{+2}^{\kappa} F_{1}\left(-\kappa, \kappa, \kappa+1,1-\frac{t}{s}\right),
$$

where ${ }_{2} F_{1}$ is the so-called Gauss hypergeometric function. For more details on ${ }_{2} F_{1}$, see Decreusefond and Üstünel (1999). 
Remark 3.2. Heuristically, the equality of (3.5) and (3.6) can be obtained as follows. If $\delta_{\{t-s\}}$ denotes the delta function at a point $t-s$, we obtain that

$$
\begin{aligned}
\frac{\mathrm{d}}{\mathrm{d} s} \int_{0}^{t} u^{\kappa}(u-s)_{+}^{\kappa} \mathrm{d} u & =\frac{\mathrm{d}}{\mathrm{d} s} \int_{0}^{a}(v+s)^{\kappa} v^{\kappa} 1_{\{0<v<t-s\}}(v) \mathrm{d} v \\
& =\kappa \int_{0}^{a}(v+s)^{\kappa-1} v^{\kappa} 1_{\{0<v<t-s\}}(v) \mathrm{d} v-\int_{0}^{a}(v+s)^{\kappa} v^{\kappa} \delta_{\{t-s\}}(v) \mathrm{d} v \\
& =\kappa \int_{s}^{t} u^{\kappa-1}(u-s)_{+}^{\kappa} \mathrm{d} u-t^{\kappa}(t-s)_{+}^{\kappa} .
\end{aligned}
$$

\section{Integrands for fractional Brownian motion on an interval}

Let $\left\{B^{\kappa}(u)\right\}_{u \in[0, a]}$ be a standard FBM with parameter $\kappa \in\left(-\frac{1}{2}, \frac{1}{2}\right)$. Let $\mathcal{E}_{a}$ denote the set of all elementary (step) functions on an interval $[0, a]$, that is, functions of the following type:

$$
f(u)=\sum_{k=1}^{n} f_{k} 1_{\left[u_{k}, u_{k+1}\right)}(u), \quad f_{k} \in \mathbb{R}, u_{k} \in[0, a] .
$$

For an elementary function $f \in \mathcal{E}_{a}$ in (4.1), define the integral with respect to the FBM $B^{\kappa}$ in a natural way by

$$
\mathcal{I}_{a}^{\kappa}(f)=\int_{0}^{a} f(u) \mathrm{d} B^{\kappa}(u)=\sum_{k=1}^{n} f_{k}\left(B^{\kappa}\left(u_{k+1}\right)-B^{\kappa}\left(u_{k}\right)\right) .
$$

It follows by Proposition 3.1 that, for $f \in \mathcal{E}_{a}$,

$$
\mathcal{I}_{a}^{\kappa}(f) \stackrel{d}{=} \sigma_{1}(\kappa) \int_{0}^{a} s^{-\kappa}\left(I_{a-}^{\kappa} u^{\kappa} f(u)\right)(s) \mathrm{d} B^{0}(s)
$$

and hence that, for all $f, g \in \mathcal{E}_{a}$,

$$
\mathrm{E}\left(\mathcal{I}_{a}^{\kappa}(f) \mathcal{I}_{a}^{\kappa}(g)\right)=\sigma_{1}(\kappa)^{2} \int_{0}^{a} s^{-2 \kappa}\left(I_{a-}^{\kappa} u^{\kappa} f(u)\right)(s)\left(I_{a-}^{\kappa} u^{\kappa} g(u)\right)(s) \mathrm{d} s .
$$

The following theorem will be used to extend the map $\mathcal{I}_{a}^{\kappa}$ on $\mathcal{E}_{a}$ to functions from $\Lambda_{a}^{\kappa}$ when $\kappa \in\left(0, \frac{1}{2}\right)$.

Theorem 4.1. For $\kappa \in\left(0, \frac{1}{2}\right)$, the class of functions $\Lambda_{a}^{\kappa}$, defined by (1.2), is a linear space with the inner product (1.4). Moreover, the set of elementary functions $\mathcal{E}_{a}$ is dense in the space $\Lambda_{a}^{\kappa}$.

We prove Theorem 4.1 in Section 8. Now let $\kappa \in\left(0, \frac{1}{2}\right)$. By Theorem 4.1, since the set of elementary functions $\mathcal{E}_{a}$ is dense in the space $\Lambda_{a}^{\kappa}$, one can extend the map $\mathcal{I}_{a}^{\kappa}$ : $\mathcal{E}_{a} \mapsto \overline{\mathrm{sp}}_{[0, a]}\left(B^{\kappa}\right)$ to the map $\mathcal{I}_{a}^{\kappa}: \Lambda_{a}^{\kappa} \mapsto \overline{\mathrm{sp}}_{[0, a]}\left(B^{\kappa}\right)$ in a classical way so that relation (4.4) continues to hold for functions $f, g \in \Lambda_{a}^{\kappa}$. Since the extended map $\mathcal{I}_{a}^{\kappa}$ is linear and 
preserves inner products, one can say that $\Lambda_{a}^{\kappa}$ is isometric to a linear subspace of $\overline{\operatorname{sp}}_{[0, a]}\left(B^{\kappa}\right)$. The question is whether this linear subspace of $\overline{\operatorname{sp}}_{[0, a]}\left(B^{\kappa}\right)$ is the space $\overline{\operatorname{sp}}_{[0, a]}\left(B^{\kappa}\right)$ itself. It is clear that $\Lambda_{a}^{\kappa}$ is isometric to $\overline{\operatorname{sp}}_{[0, a]}\left(B^{\kappa}\right)$ itself if and only if $\Lambda_{a}^{\kappa}$ is a complete inner-product space. We will show in Section 5 that $\Lambda_{a}^{\kappa}$ is not a complete inner-product space. Hence, it is not isometric to $\overline{\operatorname{sp}}_{[0, a]}\left(B^{\kappa}\right)$ itself. It is isometric to a proper linear subspace of $\overline{\operatorname{sp}}_{[0, a]}\left(B^{\kappa}\right)$ only.

Let us now show that $|\Lambda|_{a}^{\kappa}$, given by (1.3), is a linear subspace of the class of integrands $\Lambda_{a}^{\kappa}$. Suppose that the function $f$ is such that $|f| \in \Lambda_{a}^{\kappa}$ so that $f \in \Lambda_{a}^{\kappa}$ as well. Then, as in the proof of Proposition 3.1, by the Fubini's theorem we obtain

$$
\int_{0}^{a} s^{-2 \kappa}\left[\left(I_{a-}^{\kappa} u^{\kappa} f(u)\right)(s)\right]^{2} \mathrm{~d} s=\frac{\mathrm{B}(\kappa, 1-2 \kappa)}{\Gamma(\kappa)^{2}} \int_{0}^{a} \int_{0}^{a} f(u) f(v)|u-v|^{2 \kappa-1} \mathrm{~d} u \mathrm{~d} v,
$$

which shows that $|\Lambda|_{a}^{\kappa} \subset \Lambda_{a}^{\kappa}$. A linear space $|\Lambda|_{a}^{\kappa}$ becomes an inner-product space if the inner product on $|\Lambda|_{a}^{\kappa}$ is defined by $(f, g)_{|\Lambda|_{a}^{\kappa}}=(f, g)_{\Lambda_{a}^{\kappa}}$ for $f, g \in|\Lambda|_{a}^{\kappa}$. It follows by (1.4) and (4.5) that

$$
(f, g)_{|\Lambda|_{a}^{\kappa}}=\frac{\sigma_{1}(\kappa)^{2} \mathrm{~B}(\kappa, 1-2 \kappa)}{\Gamma(\kappa)^{2}} \int_{0}^{a} \int_{0}^{a} f(u) f(v)|u-v|^{2 \kappa-1} \mathrm{~d} u \mathrm{~d} v .
$$

Observe, however, that the constant in (4.6) equals $\kappa(2 \kappa+1)$ because $\left(1_{\left[0, t_{1}\right)}, 1_{\left[0, t_{2}\right)}\right)_{|\Lambda|_{a}^{\kappa}}=$ $\Gamma^{\kappa}\left(t_{1}, t_{2}\right)=\kappa(2 \kappa+1) \int_{0}^{a} \int_{0}^{a} 1_{\left[0, t_{1}\right)}(u) 1_{\left[0, t_{2}\right)}(v)|u-v|^{2 \kappa-1} \mathrm{~d} u \mathrm{~d} v$ for $t_{1}, t_{2} \in[0, a]$ by relation (3.3). In other words, the inner product on $|\Lambda|_{a}^{\kappa}$ equals (1.5) as well.

Remark 4.1. An $\mathbb{R}$-analogue of the space of integrands $|\Lambda|_{a}^{\kappa}$ is the class of functions

$$
|\Lambda|^{\kappa}=\left\{f: \mathbb{R} \mapsto \mathbb{R} \text { such that } \int_{\mathbb{R}} \int_{\mathbb{R}}|f(u)\|f(v)\| u-v|^{2 \kappa-1} \mathrm{~d} u \mathrm{~d} v<\infty\right\},
$$

for $\kappa \in\left(0, \frac{1}{2}\right)$ (see Pipiras and Taqqu 2000). It is also an inner-product space with the inner product

$$
(f, g)_{|\Lambda|^{\kappa}}=\kappa(2 \kappa+1) \int_{\mathbb{R}} \int_{\mathbb{R}} f(u) g(v)|u-v|^{2 \kappa-1} \mathrm{~d} u \mathrm{~d} v .
$$

Moreover, as shown in Pipiras and Taqqu (2000), $|\Lambda|^{\kappa}$ is a strict subspace of the class of integrands $\Lambda^{\kappa}$, given by (1.6), and the inner products (1.7) and (4.8) coincide when $f, g$ belong to the smaller class $|\Lambda|^{\kappa}$.

Remark 4.2. For $\kappa \in\left(0, \frac{1}{2}\right)$, the following useful inclusions hold:

$$
L^{2}[0, a] \subset L^{2 / 2 \kappa+1}[0, a] \subset|\Lambda|_{a}^{\kappa} \subset \Lambda_{a}^{\kappa} .
$$

The first inclusion in (4.9) is obvious since $2 / 2 \kappa+1<2$ for $\kappa>0$. According to Proposition 4.2 in Pipiras and Taqqu (2000), we have $\|f\|_{|\Lambda|^{\kappa}} \leqslant c_{\kappa}\|f\|_{L^{2 / 2 \kappa+1}(\mathbb{R})}$ for some $c_{\kappa}>0$ and all $f \in L^{2 / 2 \kappa+1}(\mathbb{R})$. This implies the second inclusion in (4.9), since

$$
\|f\|_{|\Lambda|_{a}^{\kappa}}=\left\|f 1_{[0, a]}\right\|_{|\Lambda|^{\kappa}} \leqslant c_{\kappa}\left\|f 1_{[0, a]}\right\|_{L^{2 / 2 \kappa+1}(\mathbb{R})}=c_{\kappa}\|f\|_{L^{2 / 2 \kappa+1}[0, a]} .
$$


Let us also observe that the inclusion $L^{2}[0, a] \subset|\Lambda|_{a}^{\kappa}$ is easy to verify directly. Indeed, by using the inequality $2|f(u)||f(v)| \leqslant|f(u)|^{2}+|f(v)|^{2}$ and symmetry below, we obtain

$$
\int_{0}^{a} \int_{0}^{a}|f(u)||f(v)||u-v|^{2 \kappa-1} \mathrm{~d} u \mathrm{~d} v \leqslant \int_{0}^{a} \int_{0}^{a}|f(u)|^{2}|u-v|^{2 \kappa-1} \mathrm{~d} u \mathrm{~d} v \leqslant \frac{a^{2 \kappa}}{\kappa} \int_{0}^{a}|f(u)|^{2} \mathrm{~d} u .
$$

Suppose now that $\kappa \in\left(-\frac{1}{2}, 0\right)$. Set $f(u)=u^{-\kappa}\left(I_{a-}^{-\kappa} s^{\kappa} \phi(s)\right)(u)$ for some function $\phi \in$ $L^{2}[0, a]$. It is well defined for $\phi \in L^{2}[0, a]$ because the function $s^{\kappa} \phi(s)$ is in $L^{1}[0, a]$ for $\kappa>-\frac{1}{2}$ by the Hölder's inequality. Property (2.5) then implies

$$
s^{-\kappa}\left(I_{a-}^{\kappa} u^{\kappa} f(u)\right)(s)=s^{-\kappa}\left(\mathscr{D}_{a-}^{-\kappa} u^{\kappa} u^{-\kappa}\left(I_{a-}^{-\kappa} z^{\kappa} \phi(z)\right)(u)\right)(s)=\phi(s)
$$

and hence that

$$
\int_{0}^{a} s^{-2 \kappa}\left[\left(I_{a-}^{\kappa} u^{\kappa} f(u)\right)(s)\right]^{2} \mathrm{~d} s=\int_{0}^{a} \phi(s)^{2} \mathrm{~d} s<\infty .
$$

Based on this observation and relations (4.3) and (4.4), it is natural to introduce the class of functions $\Lambda_{a}^{\kappa}$ given by (1.8). The following result is analogous to Theorem 4.1 and deals with the case $\kappa \in\left(-\frac{1}{2}, 0\right)$.

Theorem 4.2. For $\kappa \in\left(-\frac{1}{2}, 0\right)$, the class of functions $\Lambda_{a}^{\kappa}$, defined by (1.8), is a linear space with the inner product (1.9) or (1.10). Moreover, the set of elementary functions $\mathcal{E}_{a}$ is dense in the space $\Lambda_{a}^{\kappa}$.

We prove Theorem 4.2 in Section 8. By Theorem 4.2, since the set of elementary functions $\mathcal{E}_{a}$ is dense in $\Lambda_{a}^{\kappa}$, one can extend the map $\mathcal{I}_{a}^{\kappa}: \mathcal{E}_{a} \mapsto \overline{\operatorname{sp}}_{[0, a]}\left(B^{\kappa}\right)$ to the map $\mathcal{I}_{a}^{\kappa}: \Lambda_{a}^{\kappa} \mapsto \overline{\operatorname{sp}}_{[0, a]}\left(B^{\kappa}\right)$ so that it is linear and preserves inner products.

The space $\Lambda_{a}^{\kappa}$ can be viewed as a class of integrands for FBM on an interval $[0, a]$. It is also isometric to a linear subspace of $\overline{\operatorname{sp}}_{[0, a]}\left(B^{\kappa}\right)$. Is this linear subspace the space $\overline{\operatorname{sp}}_{[0, a]}\left(B^{\kappa}\right)$ itself? Or, equivalently, is the space of functions $\Lambda_{a}^{\kappa}$ a complete inner-product space? The answer is an obvious yes. Indeed, suppose that $\left\{f_{n}\right\}_{n \geqslant 1}$ is a Cauchy sequence in $\Lambda_{a}^{k}$. Then the sequence of functions $\phi_{f_{n}}$, associated with the functions $f_{n}$ by definition (1.8), is Cauchy in $L^{2}[0, a]$. Since the space $L^{2}[0, a]$ is complete, there is a function $\phi \in L^{2}[0, a]$ such that $\phi_{f_{n}} \rightarrow \phi$ in $L^{2}[0, a]$. If $f(u)=u^{-\kappa}\left(I_{a-}^{-\kappa} s^{k} \phi(s)\right)(u)$, then $f_{n} \rightarrow f$ in $\Lambda_{a}^{\kappa}$ since $\phi_{f_{n}} \rightarrow \phi$ in $L^{2}[0, a]$. This shows the completeness of $\Lambda_{a}^{\kappa}$.

Remark 4.3. An $\mathbb{R}$-analogue of the space of integrands $\Lambda_{a}^{\kappa}$ with $\kappa \in\left(-\frac{1}{2}, 0\right)$ is the class of functions

$$
\Lambda^{\kappa}=\left\{f: \exists \phi_{f} \in L^{2}(\mathbb{R}) \text { such that } f=I_{-}^{-\kappa} \phi_{f}\right\}
$$

(see Pipiras and Taqqu 2000). The class $\Lambda^{\kappa}$ is an inner-product space with the inner product 


$$
\begin{aligned}
(f, g)_{\Lambda^{\kappa}} & =\frac{\Gamma(\kappa+1)^{2}}{c_{1}(\kappa)^{2}} \int_{\mathbb{R}}\left(\mathbf{D}_{-}^{-\kappa} f\right)(s)\left(\mathbf{D}_{-}^{-\kappa} g\right)(s) \mathrm{d} s \\
& =\frac{\Gamma(\kappa+1)^{2}}{c_{1}(\kappa)^{2}} \int_{\mathbb{R}} \phi_{f}(s) \phi_{g}(s) \mathrm{d} s,
\end{aligned}
$$

where $\mathbf{D}_{-}^{-\kappa}, \kappa \in\left(-\frac{1}{2}, 0\right)$, is the so-called Marchaud fractional derivative of order $-\kappa$ which has the property $\mathbf{D}_{-}^{-\kappa} I_{-}^{-\kappa} \phi=\phi$ for $\kappa \in\left(-\frac{1}{2}, 0\right)$ and $\phi \in L^{2}(\mathbb{R})$. Observe that, when $\kappa \in$ $\left(-\frac{1}{2}, 0\right)$, both the space $\Lambda^{\kappa}$ and the space $\Lambda_{a}^{\kappa}$ are built up by exploiting the same idea.

Remark 4.4. We emphasize again that the inner-product space $\Lambda_{a}^{\kappa}$ is complete if $\kappa \in\left(-\frac{1}{2}, 0\right)$, and incomplete if $\kappa \in\left(0, \frac{1}{2}\right)$. The proof in Section 5 below and the proof of completeness preceding Remark 4.3 show that this difference in completeness is a consequence of the following two facts:

(a) If $\kappa \in\left(-\frac{1}{2}, 0\right)$, then the equation

$$
s^{-\kappa}\left(I_{a-}^{\kappa} u^{\kappa} f(u)\right)(s)=s^{-\kappa}\left(\mathscr{D}_{a-}^{-\kappa} u^{\kappa} f(u)\right)(s)=\phi(s)
$$

has a solution $f(u)=u^{-\kappa}\left(I_{a-}^{-\kappa} s^{\kappa} \phi(s)\right)(u)$ for every $\phi \in L^{2}[0, a]$.

(b) When $\kappa \in\left(0, \frac{1}{2}\right)$, however, there are functions $\phi \in L^{2}[0, a]$ for which the equation

$$
s^{-\kappa}\left(I_{a-}^{\kappa} u^{\kappa} f(u)\right)(s)=\phi(s)
$$

is not solvable. The idea here is that, since $I_{a-}^{k}$ is the integral operator, the left-hand side of (4.15) must satisfy some smoothness conditions (for example, one can take its weighted fractional derivative $\left(\mathcal{D}_{a-}^{\kappa} s^{\kappa}\right)$ ) whereas such smoothness conditions need not hold for a general $\phi \in L^{2}[0, a]$.

\section{Incompleteness of classes of integrands when $0<\kappa<\frac{1}{2}$}

The goal of this section is to show that neither the space $|\Lambda|_{a}^{\kappa}$ nor the space $\Lambda_{a}^{\kappa}$ is a complete inner-product space when $\kappa \in\left(0, \frac{1}{2}\right)$. We will give a proof by first providing an equivalent criterion for the completeness and then showing that it does not hold. We begin with a number of lemmas which will be required. The parameter $\kappa$ is in the range $\left(0, \frac{1}{2}\right)$ throughout.

Lemma 5.1. Let $0 \leqslant c<b \leqslant a$ and $\kappa \in\left(0, \frac{1}{2}\right)$. Then there is a function $f_{c, b}$ such that

$$
s^{-\kappa}\left(I_{a-}^{\kappa} u^{\kappa} f_{c, b}(u)\right)(s)=1_{[c, b)}(s), \quad \text { for all } 0 \leqslant s \leqslant a .
$$

Proof. Heuristically, by solving equation (5.1), we obtain that

$$
f_{c, b}(u)=u^{-\kappa}\left(I_{a-}^{-\kappa} s^{\kappa} 1_{[c, b)}(s)\right)(u)=u^{-\kappa}\left(\mathcal{D}_{a-}^{\kappa} s^{\kappa} 1_{[c, b)}(s)\right)(u) .
$$

To show that this function indeed satisfies (5.1), we may assume by linearity that $c=0$. We may also show (5.1) for $s<b$ only, since for $s \geqslant b$ it is obvious (both sides are zero). As in (3.5) and (3.6) (see also Remark 3.2 for heuristics), we have that 


$$
-\Gamma(1-\kappa)\left(\mathcal{D}_{a-}^{\kappa} s^{\kappa} 1_{[c, b)}(s)\right)(u)=\kappa \int_{u}^{b} s^{\kappa-1}(s-u)^{-\kappa} \mathrm{d} s-b^{\kappa}(b-u)_{+}^{-\kappa} .
$$

Then, for $s<b$,

$$
\begin{aligned}
-\Gamma(1-\kappa) & \Gamma(\kappa) s^{-\kappa}\left(I_{a-}^{\kappa} u^{\kappa} u^{-\kappa}\left(\mathcal{D}_{a-}^{\kappa} z^{\kappa} 1_{[c, b)}(z)\right)(u)\right)(s) \\
& =s^{-\kappa} \int_{s}^{a}\left\{\kappa \int_{u}^{b} z^{\kappa-1}(z-u)^{-\kappa} \mathrm{d} z-b^{\kappa}(b-u)_{+}^{-\kappa}\right\}(u-s)^{\kappa-1} \mathrm{~d} u \\
& =s^{-\kappa} \kappa \int_{s}^{b} \mathrm{~d} z z^{\kappa-1} \int_{s}^{z}(z-u)^{-\kappa}(u-s)^{\kappa-1} \mathrm{~d} u-s^{-\kappa} b^{\kappa} \int_{s}^{b}(b-u)_{+}^{-\kappa}(u-s)^{\kappa-1} \mathrm{~d} u \\
& =\mathrm{B}(1-\kappa, \kappa)\left(\kappa s^{-\kappa} \int_{s}^{b} z^{\kappa-1} \mathrm{~d} z-s^{-\kappa} b^{\kappa}\right)=-\Gamma(1-\kappa) \Gamma(\kappa),
\end{aligned}
$$

since $\int_{s}^{z}(z-u)^{-\kappa}(u-s)^{\kappa-1} \mathrm{~d} u=\mathrm{B}(1-\kappa, \kappa)=\Gamma(1-\kappa) \Gamma(\kappa)$. This shows (5.1).

The proof of the following lemma is similar to that of Lemma 5.6 in Pipiras and Taqqu (2000). We include it for the reader's convenience.

Lemma 5.2. Let $\kappa \in\left(0, \frac{1}{2}\right)$. The inner-product space $\Lambda_{a}^{\kappa}$ is complete if and only if, for every $\phi \in L^{2}[0, a]$, there is a function $f_{\phi} \in \Lambda_{a}^{\kappa}$ such that

$$
s^{-\kappa}\left(I_{a-}^{\kappa} u^{\kappa} f_{\phi}(u)\right)(s)=\phi(s) \text { almost everywhere } \mathrm{d} s .
$$

Proof. Suppose that the inner-product space $\Lambda_{a}^{\kappa}$ is complete and let $\phi \in L^{2}[0, a]$. There is a sequence of elementary functions $\phi_{n}$ such that $\phi_{n} \rightarrow \phi$ in $L^{2}[0, a]$. By Lemma 5.1, we can express the elementary functions $\phi_{n}$ as $\phi_{n}=s^{-\kappa}\left(I_{a-}^{\kappa} u^{\kappa} f_{n}(u)\right)(s)$, for some $f_{n} \in \Lambda_{a}^{\kappa}$. Since the sequence $\left\{\phi_{n}\right\}_{n \geqslant 1}$ is Cauchy in $L^{2}[0, a]$, the sequence $\left\{f_{n}\right\}_{n \geqslant 1}$ is Cauchy in $\Lambda_{a}^{k}$. Then the completeness of $\Lambda_{a}^{\kappa}$ implies that there is $f \in \Lambda_{a}^{\kappa}$ such that $\phi_{n}=$ $s^{-\kappa}\left(I_{a-}^{\kappa} u^{\kappa} f_{n}(u)\right)(s) \rightarrow s^{-\kappa}\left(I_{a-}^{\kappa} u^{\kappa} f(u)\right)(s)$ in $L^{2}[0, a]$. Since $\phi_{n} \rightarrow \phi$ in $L^{2}[0, a]$ as well, relation (5.3) holds with $f_{\phi}=f$.

Conversely, suppose that (5.3) holds and let $\left\{f_{n}\right\}_{n \geqslant 1}$ be a Cauchy sequence in $\Lambda_{a}^{\kappa}$. Then the sequence $\phi_{n}(s)=s^{-\kappa}\left(I_{a-}^{\kappa} u^{\kappa} f_{n}(u)\right)(s)$ is Cauchy in $L^{2}[0, a]$. Since $L^{2}[0, a]$ is complete, there is a $\phi \in L^{2}[0, a]$ such that $\phi_{n} \rightarrow \phi$ in $L^{2}[0, a]$. By the assumption, there is an $f_{\phi} \in \Lambda^{\kappa}$ a such that (5.3) holds. Since $\phi_{n} \rightarrow \phi$ in $L^{2}[0, \mathrm{a}]$ implies $\mathrm{f}_{\mathrm{n}} \rightarrow \mathrm{f}_{\phi}$ in $\Lambda_{a}^{\kappa}$, the space $\Lambda_{a}^{\kappa}$ is complete.

Lemma 5.3. Let $\kappa \in\left(0, \frac{1}{2}\right)$. There are continuous functions $\psi$ on $[0, a]$ such that the equation

$$
\left(I_{a-}^{\kappa} g\right)(s)=\psi(s) \text { a.e. } \mathrm{d} s \text { on }[0, a]
$$

has no solution in $g \in L^{1}[0, a]$.

Proof. The proof of the lemma is by contradiction. Suppose that (5.4) has a solution $g_{\psi} \in$ $L^{1}[0, a]$ for any $\psi \in L^{2}[0, a]$. By $(2.4)$, 


$$
g_{\psi}(u)=-\frac{1}{\Gamma(1-\kappa)} \frac{\mathrm{d}}{\mathrm{d} u} \int_{u}^{a} \psi(s)(s-u)_{+}^{-\kappa} \mathrm{d} s, \quad u \in(0, a)
$$

(see also Samko et al. 1993, Section 2.1). Since $g_{\psi}(u)$ is expressed as a derivative, the function

$$
U_{\psi}(u)=\int_{0}^{a} \psi(s)(s-u)_{+}^{-\kappa} \mathrm{d} s=\int_{\mathbb{R}} \psi(s+u) 1_{\{0<s+u<a\}} s_{+}^{-\kappa} \mathrm{d} s, \quad u \in(0, a),
$$

is differentiable almost everywhere on $(0, a)$. However, as shown in Lemma 5.7 of Pipiras and Taqqu (2000), there are functions $\psi \in L^{2}[0, a]$ such that $U_{\psi}$ is not differentiable on a set of positive Lebesgue measure. For example, when $a=1$ (otherwise, use appropriate scaling), for the function $\psi$ we can take the real or imaginary part of

$$
\psi^{*}(u)=c_{0} \sum_{n=1}^{\infty} b^{-p n} \mathrm{e}^{\mathrm{i} b^{n} u},
$$

where $b>1,0<p<\kappa$ and $c_{0}=(\Gamma(1-\kappa))^{-1} \mathrm{e}^{-\mathrm{i} \pi(1-\kappa) / 2}$. Indeed, for $v \in(0,1)$, we have

$$
\begin{aligned}
U_{\psi^{*}}(v) & =c_{0} \int_{0}^{1-v} s_{+}^{-\kappa}\left[\sum_{n=1}^{\infty} b^{-p n} \mathrm{e}^{\mathrm{i} b^{n} s} \mathrm{e}^{\mathrm{i} b^{n} v}\right] \mathrm{d} s=c_{0} \sum_{n=1}^{\infty} b^{-p n}\left[\int_{0}^{1-v} \mathrm{e}^{\mathrm{i} b^{n} s} s_{+}^{-\kappa} \mathrm{d} s\right] \mathrm{e}^{\mathrm{i} b^{n} v} \\
& =c_{0} \sum_{n=1}^{\infty} b^{-p n}\left[\int_{0}^{\infty} \mathrm{e}^{\mathrm{i} b^{n} s} s_{+}^{-\kappa} \mathrm{d} s\right] \mathrm{e}^{\mathrm{i} b^{n} v}-c_{0} \sum_{n=1}^{\infty} b^{-p n}\left[\int_{1-v}^{\infty} \mathrm{e}^{\mathrm{i} b^{n} s} s_{+}^{-\kappa} \mathrm{d} s\right] \mathrm{e}^{\mathrm{i} b^{n} v} \\
& =: y_{1}(v)+y_{2}(v) .
\end{aligned}
$$

As indicated in Pipiras and Taqqu (2000), the idea then is to show that the function $y_{1}$ is not differentiable on $[0,1]$ and that the function $y_{2}$ is differentiable on $\left[0, \frac{1}{2}\right]$. By making a change of variables $b^{n} s=z$ in $y_{1}$, we obtain that

$$
y_{1}(v)=\sum_{n=1}^{\infty} b^{-(p-\kappa+1) n} \mathrm{e}^{\mathrm{i} b^{n} v} .
$$

Since $b^{-(p-\kappa+1)} b=b^{\kappa-p}>1$, the function $y_{1}$ is a particular case of the well-known Weierstrass function whose real and imaginary parts are nowhere differentiable functions. One can show that the function $y_{2}$ is differentiable on $\left[0, \frac{1}{2}\right]$ by using standard results from real analysis (for details, see Pipiras and Taqqu 2000). Finally, since the function (5.5) is continuous, we obtain the result.

By combining the previous lemmas, we obtain:

Theorem 5.1. Let $\kappa \in\left(0, \frac{1}{2}\right)$. The inner-product spaces $\Lambda_{a}^{\kappa}$ and $|\Lambda|_{a}^{\kappa}$ are not complete.

Proof. For the inner-product space $\Lambda_{a}^{\kappa}$ this theorem follows from Lemmas 5.2 and 5.3. Indeed, by Lemma 5.3, there is a continuous function $\psi$ such that equation (5.4) has no 
solution in $g$. Then the function $\phi(s)=s^{-\kappa} \psi(s)$ is in $L^{2}[0, a]$, since $\kappa<\frac{1}{2}$, and equation (5.3) has no solution in $f$. Thus, by Lemma 5.2, the inner-product space $\Lambda_{a}^{\kappa}$ is not complete.

We now turn to the inner-product space $|\Lambda|_{a}^{\kappa}$. Since $\Lambda_{a}^{\kappa}$ is not complete and the set of elementary (step) functions $\mathcal{E}^{a}$ is dense in $\Lambda_{a}^{\kappa}$, there is a Cauchy sequence $\left\{f_{n}\right\}_{n \geqslant 1} \subset \mathcal{E}^{a}$ which does not converge in $\Lambda_{a}^{\kappa}$. Since $\mathcal{E}^{a} \subset|\Lambda|_{a}^{\kappa}$ and $(g, h)_{|\Lambda|_{a}^{\kappa}}=(g, h)_{\Lambda_{a}^{\kappa}}$, for $g, h \in \mathcal{E}^{a}$, the sequence $\left\{f_{n}\right\}_{n \geqslant 1} \subset \mathcal{E}^{a}$ is also Cauchy in $|\Lambda|_{a}^{\kappa}$. If $|\Lambda|_{a}^{\kappa}$ is complete, there is an $f \in|\Lambda|_{a}^{\kappa}$ such that $f_{n} \rightarrow f$ in $|\Lambda|_{a}^{\kappa}$. But then $f_{n} \rightarrow f$ in $\Lambda_{a}^{\kappa}$ as well because $|\Lambda|_{a}^{\kappa} \subset \Lambda_{a}^{\kappa}$ and $(g, h)_{|\Lambda|_{a}^{\kappa}}=(g, h)_{\Lambda_{a}^{\kappa}}$, for $g, h \in|\Lambda|_{a}^{\kappa}$. Since this is a contradiction, we obtain that the innerproduct space $|\Lambda|_{a}^{\kappa}$ is not complete.

\section{The case of the positive half-axis}

As mentioned in Section 1, the results on incompleteness of classes of integrands are also valid when $a=\infty$ and $\kappa \in\left(0, \frac{1}{2}\right)$. When $\kappa \in\left(0, \frac{1}{2}\right)$, by using the scheme described in Section 4 , one can construct the following two classes of integrands on $[0, \infty)$ :

$$
\Lambda_{\infty}^{\kappa}=\left\{f:[0, \infty) \mapsto \mathbb{R} \text { such that } \int_{0}^{\infty}\left[s^{-\kappa}\left(I_{-}^{\kappa} u^{\kappa} f(u)\right)(s)\right]^{2} \mathrm{~d} s<\infty\right\},
$$

where $\left(I_{-}^{\kappa} \phi\right)(s)=(\Gamma(\kappa))^{-1} \int_{\mathbb{R}} \phi(u)(u-s)_{+}^{\kappa-1} \mathrm{~d} u, s>0$, is a fractional integral of order $\kappa$; and

$$
|\Lambda|_{\infty}^{\kappa}=\left\{f:[0, \infty) \mapsto \mathbb{R} \text { such that } \int_{0}^{\infty} \int_{0}^{\infty}|f(u)||f(v)||u-v|^{2 \kappa-1} \mathrm{~d} u \mathrm{~d} v<\infty\right\} .
$$

As in the case $a>0$, the classes of integrands $\Lambda_{\infty}^{\kappa}$ and $|\Lambda|_{\infty}^{\kappa}$ are inner-product spaces with the inner products

$$
(f, g)_{\Lambda_{\infty}^{\kappa}}=\frac{\pi \kappa(2 \kappa+1)}{\Gamma(1-2 \kappa) \sin \pi \kappa} \int_{0}^{\infty} s^{-2 \kappa}\left(I_{-}^{\kappa} u^{\kappa} f(u)\right)(s)\left(I_{-}^{\kappa} u^{\kappa} g(u)\right)(s) \mathrm{d} s
$$

and

$$
(f, g)_{|\Lambda|_{\infty}^{\kappa}}=\kappa(2 \kappa+1) \int_{0}^{\infty} \int_{0}^{\infty} f(u) g(v)|u-v|^{2 \kappa-1} \mathrm{~d} u \mathrm{~d} v,
$$

respectively. We also have that $|\Lambda|_{\infty}^{\kappa} \subset \Lambda_{\infty}^{\kappa}$ and that the corresponding inner products are equal for functions $f, g$ belonging to the smaller class $|\Lambda|_{\infty}^{\kappa}$.

Corollary 6.1. Let $\kappa \in\left(0, \frac{1}{2}\right)$. The inner-product spaces $\Lambda_{\infty}^{\kappa}$ and $|\Lambda|_{\infty}^{\kappa}$ are not complete.

Proof. We will show by contradiction that the inner-product space $\Lambda_{\infty}^{\kappa}$ is not complete. Suppose that $\Lambda_{\infty}^{\kappa}$ is complete. By Theorem 5.1, the inner-product space $\Lambda_{a}^{\kappa}$ is not complete. Hence, there is a Cauchy sequence $g_{n}$ of functions in $\Lambda_{a}^{\kappa}$ such that it does not converge. Since $\left(I_{-}^{\kappa} f 1_{[0, a]}\right)(s)=\left(I_{a-}^{\kappa} f\right)(s) 1_{[0, a]}(s)$, for any $f \in L^{1}[0, a]$ and $s>0$, the sequence of functions $g_{n} 1_{[0, a]}$ is also Cauchy in $\Lambda_{\infty}^{\kappa}$. The completeness of $\Lambda_{\infty}^{\kappa}$ then implies that there is a function $g \in \Lambda_{\infty}^{\kappa}$ such that $g_{n} 1_{[0, a]} \rightarrow g$ in $\Lambda_{\infty}^{\kappa}$, that is, $s^{-\kappa}\left(I_{-}^{\kappa} u^{\kappa} g_{n}(u) 1_{[0, a]}(u)\right)(s)$ converges to $s^{-\kappa}\left(I_{-}^{\kappa} u^{\kappa} g(u)\right)(s)$ in the $L^{2}(\mathbb{R})$ sense. Since $\left(I_{-}^{\kappa} u^{\kappa} g_{n}(u) 1_{[0, a]}(u)\right)(s)=0$, for $s>a$, we 
obtain that $\left(I_{-}^{\kappa} u^{\kappa} g(u)\right)(s)=0$, for $s>a$, as well. By Lemma 6.1 below, $g(u)=0$ a.e. for $u>a$, that is, $g(u)=g(u) 1_{[0, a]}(u)$ a.e. for $u>0$. Since

$$
\begin{aligned}
\int_{0}^{a}\left[s^{-\kappa}\left(I_{a-}^{\kappa} u^{\kappa}\left(g_{n}(u)-g(u)\right)\right)(s)\right]^{2} \mathrm{~d} s & =\int_{0}^{\infty}\left[s^{-\kappa}\left(I_{-}^{\kappa} u^{\kappa}\left(g_{n}(u) 1_{[0, a]}(u)-g(u) 1_{[0, a]}\right)\right)(s)\right]^{2} \mathrm{~d} s \\
& =\int_{0}^{\infty}\left[s^{-\kappa}\left(I_{-}^{\kappa} u^{\kappa}\left(g_{n}(u) 1_{[0, a]}(u)-g(u)\right)\right)(s)\right]^{2} \mathrm{~d} s \rightarrow 0,
\end{aligned}
$$

as $n \rightarrow \infty, g_{n} \rightarrow g$ in $\Lambda_{a}^{\kappa}$, which is a contradiction. Hence, the inner-product space $\Lambda_{\infty}^{\kappa}$ is not complete.

The inner-product space $|\Lambda|_{\infty}^{\kappa}$ is not complete either, by arguments similar to those in the proof of Theorem 5.1.

Lemma 6.1. Let $\kappa \in\left(0, \frac{1}{2}\right), a>0$ be fixed and $g \in \Lambda_{\infty}^{\kappa}$. If, for $s>a,\left(I_{-}^{\kappa} u^{\kappa} g(u)\right)(s)=0$ a.e. $\mathrm{d} s$, then, for $u>a, g(u)=0$ a.e. $\mathrm{d} u$.

Proof. Let $\psi_{c, b}(s)=(\Gamma(1-\kappa))^{-1}\left((s-c)_{+}^{-\kappa}-(s-b)_{+}^{-\kappa}\right), s \in \mathbb{R}$, be a function with $a<c<b$ and observe that it vanishes for $s \leqslant c$. Now define the operator $I_{+}^{\kappa}$ by

$$
\left(I_{+}^{\kappa} \psi\right)(u)=\frac{1}{\Gamma(\kappa)} \int_{\mathbb{R}} \psi(s)(u-s)_{+}^{\kappa-1} \mathrm{~d} s, \quad s \in \mathbb{R} .
$$

Heuristically, by changing the order of integration below, we obtain that

$$
\begin{aligned}
0 & =\int_{a}^{\infty}\left(I_{-}^{\kappa} u^{\kappa} g(u)\right)(s) \psi_{c, b}(s) \mathrm{d} s=\frac{1}{\Gamma(\kappa)} \int_{a}^{\infty}\left\{\int_{a}^{\infty} u^{\kappa} g(u)(u-s)_{+}^{\kappa-1} \mathrm{~d} u\right\} \psi_{c, b}(s) \mathrm{d} s \\
& =\frac{1}{\Gamma(\kappa)} \int_{a}^{\infty} u^{\kappa} g(u)\left\{\int_{a}^{\infty} \psi_{c, b}(s)(s-u)_{-}^{\kappa-1} \mathrm{~d} s\right\} \mathrm{d} u=\int_{a}^{\infty} u^{\kappa} g(u)\left(I_{+}^{\kappa} \psi_{c, b}\right)(u) \mathrm{d} u
\end{aligned}
$$

and

$$
0=\int_{a}^{\infty} u^{\kappa} g(u) 1_{(c, b]}(u) \mathrm{d} u
$$

since, by Lemma 6.2 below, the function $\psi_{c, b}$ has the property $I_{+}^{\kappa} \psi_{c, b}=1_{(c, b]}$. One way to prove relation (6.2) is to justify the change in the order of integration in (6.1) and show that

$$
\int_{a}^{\infty} \int_{a}^{\infty}\left|u^{\kappa} g(u)\right|\left|\psi_{c, b}(s)\right|(u-s)_{+}^{\kappa-1} \mathrm{~d} u \mathrm{~d} s<\infty .
$$

This can be done for almost every $b$. As in the proof of Lemma 5.4 in Pipiras and Taqqu (2000), by using the relation $\left|\psi_{c, b}\right|=\psi_{c, b}-2 \psi_{c, b} 1_{(b, \infty)}$, we obtain that 


$$
\begin{aligned}
&(\Gamma(\kappa))^{-1} \int_{a}^{\infty}\left|\psi_{c, b}(s)\right|(u-s)_{+}^{\kappa-1} \mathrm{~d} s \\
& \leqslant\left(I_{+}^{\kappa}\left|\psi_{c, b}\right|\right)(u) \\
&=\left(I_{+}^{\kappa} \psi_{c, b}\right)(u)-2\left(I_{+}^{\kappa} \psi_{c, b} 1_{(b, \infty)}\right)(u) \\
&=\left(I_{+}^{\kappa} \psi_{c, b}\right)(u)-2\left(I_{+}^{\kappa} \psi_{c, b} 1_{(b, \infty)}\right)(u) 1_{(b, \infty)}(u) \\
&=\left(I_{+}^{\kappa} \psi_{c, b}\right)(u)-2\left(I_{+}^{\kappa} \psi_{c, b}\right)(u) 1_{(b, \infty)}(u)+2\left(I_{+}^{\kappa} \psi_{c, b} 1_{(c, b)}\right)(u) 1_{(b, \infty)}(u) \\
&= 1_{(c, b]}(u)-21_{(c, b]}(u) 1_{(b, \infty)}(u) \\
&+2(\Gamma(\kappa) \Gamma(1-\kappa))^{-1} \int_{c}^{b}(s-c)_{+}^{-\kappa}(u-s)_{+}^{\kappa-1} \mathrm{~d} s 1_{(b, \infty)}(u) \\
& \leqslant 1_{(c, b]}(u)+2(\Gamma(\kappa) \Gamma(1-\kappa))^{-1} \int_{c}^{b}(s-c)_{+}^{-\kappa} \mathrm{d} s(u-b)_{+}^{\kappa-1} \\
&= 1_{(c, b]}(u)+c_{\kappa}(b-c)^{1-\kappa}(u-b)_{+}^{\kappa-1} .
\end{aligned}
$$

It is then enough to verify that

$$
\int_{a}^{\infty}\left|u^{\kappa} g(u)\right| 1_{(c, b]}(u) \mathrm{d} u<\infty
$$

and

$$
\int_{a}^{\infty}\left|u^{\kappa} g(u)\right|(u-b)_{+}^{\kappa-1} \mathrm{~d} u<\infty .
$$

Since $g \in \Lambda_{\infty}^{\kappa}$ implies that $\left(I_{-}^{\kappa} u^{\kappa} g(u)\right)(s)$ is well defined a.e. for $s>a$, that is,

$$
\text { for } s>a, \quad \int_{a}^{\infty}\left|u^{\kappa} g(u)\right|(u-s)_{+}^{\kappa-1} \mathrm{~d} u<\infty \text { a.e. } \mathrm{d} s,
$$

equality (6.3) follows for any $a<c<b$ (we implicitly assume that integration is in the Lebesgue sense everywhere). If $b$ is such that (6.5) holds, then we immediately obtain (6.4) and hence relation (6.2) is valid. However, if (6.5) does not hold for $b$, it holds for some $b_{n}$ such that $b_{n} \rightarrow b$. Then (6.2) with $b$ follows directly by letting $n \rightarrow \infty$ in

$$
0=\int_{a}^{\infty} u^{\kappa} g(u) 1_{\left(c, b_{n}\right]}(u) \mathrm{d} u .
$$

Finally, by approximating the set $\{u: a<u<n\} \cap\left\{u: u^{\kappa} g(u)>0\right\}$ by unions of disjoint intervals and letting $n \rightarrow \infty$, it follows from (6.2) that $u^{\kappa} g(u) \leqslant 0$ a.e. $\mathrm{d} u$ for $u>a$ and, by symmetry, that $u^{\kappa} g(u) \geqslant 0$ a.e. for $u>a$. Hence, $u^{\kappa} g(u)=0$ a.e. $\mathrm{d} u$ for $u>a$ or $g(u)=0$ a.e. $\mathrm{d} u$ for $u>a$.

Lemma 6.2. Let $c<b$ be real numbers and $\kappa \in(0,1)$. Then the function 


$$
\psi_{c, b}(s)=(\Gamma(1-\kappa))^{-1}\left((s-c)_{+}^{-\kappa}-(s-b)_{+}^{-\kappa}\right)
$$

satisfies the equation

$$
\left(I_{+}^{\kappa} \psi_{c, b}\right)(u)=1_{(c, b]}(u), \quad u \in \mathbb{R} .
$$

Proof. By the definition of $I_{+}^{\kappa}$ (see the proof above), we have to verify that, for $u \in \mathbb{R}$,

$$
\begin{aligned}
J_{1}(u) & :=\int_{\mathbb{R}}\left((s-c)_{+}^{-\kappa}-(s-b)_{+}^{-\kappa}\right)(u-s)_{+}^{\kappa-1} \mathrm{~d} s \\
& =\Gamma(\kappa) \Gamma(1-\kappa) 1_{[c, b)}(u)=: J_{2}(u) .
\end{aligned}
$$

If $u \leqslant c$, then $J_{1}(u)=J_{2}(u)=0$. If $u>c$, we use the following identity, valid for $t>0$ :

$$
\begin{aligned}
\int_{0}^{t} v^{-\kappa}(t-v)^{\kappa-1} \mathrm{~d} v & =t^{-\kappa+\kappa-1+1} \int_{0}^{1} s^{-\kappa}(1-s)^{\kappa-1} \mathrm{~d} s=\mathrm{B}(\kappa, 1-\kappa) \\
& =\frac{\Gamma(\kappa) \Gamma(1-\kappa)}{\Gamma(\kappa+1-\kappa)}=\Gamma(\kappa) \Gamma(1-\kappa) .
\end{aligned}
$$

If $c<u \leqslant b$, then

$$
J_{1}(u)=\int_{c}^{u}(s-c)_{+}^{-\kappa}(u-s)_{+}^{\kappa-1} \mathrm{~d} s=\int_{0}^{u-c} v^{-\kappa}(u-c-v)^{\kappa-1} \mathrm{~d} v=\Gamma(\kappa) \Gamma(1-\kappa),
$$

which is also $J_{2}(u)$. In the case when $b<u$, we show in a similar way that

$$
J_{1}(u)=\int_{c}^{u}(s-c)_{+}^{-\kappa}(u-s)_{+}^{\kappa-1} \mathrm{~d} s-\int_{b}^{u}(s-b)_{+}^{-\kappa}(u-s)_{+}^{\kappa-1} \mathrm{~d} s=0=J_{2}(u) .
$$

Remark 6.1. The result of Lemma 6.2 should not be surprising because of the relation $\psi_{c, b}=I_{+}^{-\kappa} 1_{(c, b]}$, where the $\kappa$-fractional derivative operator $I_{+}^{-\kappa}$, defined as

$$
\left(I_{+}^{-\kappa} f\right)(s)=\frac{1}{\Gamma(1-\kappa)} \frac{\mathrm{d}}{\mathrm{d} s} \int_{-\infty}^{s} f(u)(s-u)_{+}^{-\kappa} \mathrm{d} u, \quad s \in \mathbb{R},
$$

is the inverse of $I_{+}^{\kappa}$ (there exist other definitions for an inverse of $I_{+}^{\kappa}-$ see, for example, Section 5 in Samko et al. 1993).

\section{Prediction of fractional Brownian motion}

The prediction problem for FBM is to find an explicit expression for the conditional expectation $X=\mathrm{E}\left(B^{\kappa}(t) \mid B^{\kappa}(s), s \in[0, a]\right)$ with some fixed $0<a<t$. As already indicated in the Introduction, we have $X \in \overline{\mathrm{sp}}_{[0, a]}\left(B^{\kappa}\right)$ and therefore we expect $X=\int_{0}^{a} f(u) \mathrm{d} B^{\kappa}(u)$ for some function $f$. When $\kappa \in\left(-\frac{1}{2}, 0\right)$, since the class of integrands $\Lambda_{a}^{\kappa}$ in (1.8) is complete, we know that the function $f$ exists and belongs to $\Lambda_{a}^{\kappa}$. When $\kappa \in\left(0, \frac{1}{2}\right)$, however, since the class 
$\Lambda_{a}^{\kappa}$ in (1.2) is not complete, we may only hope that there is such a function $f$ which belongs to $\Lambda_{a}^{\kappa}$.

In this section, we will show that the above function $f$ exists not only for $\kappa \in\left(-\frac{1}{2}, 0\right)$ but also for $\kappa \in\left(0, \frac{1}{2}\right)$. We will also determine this function explicitely by first providing its heuristic derivation which uses fractional integration ideas and then verifying rigorously that the function obtained is indeed the right one. When $\kappa \in\left(0, \frac{1}{2}\right)$, the prediction formula was obtained by Gripenberg and Norros (1996). Our approach is different from that of Gripenberg and Norros, where fractional integration is not used, and it also covers the case $\kappa \in\left(-\frac{1}{2}, 0\right)$.

The prediction formula for FBM can be heuristically derived as follows. We shall repeatedly use the (heuristic) fractional integration 'rule' $I^{\alpha} I^{\beta}=I^{\alpha+\beta}$ for $\alpha, \beta \in \mathbb{R}$, where $I^{\alpha}$ is some fractional integral or derivative operator. For $s \in[0, t]$, set

$$
B^{0}(s)=\int_{0}^{t} u^{-\kappa}\left(I_{t-}^{-\kappa} z^{\kappa} 1_{[0, s)}(z)\right)(u) \mathrm{d} B^{\kappa}(u) .
$$

Since $v^{-\kappa}\left(I_{t-}^{\kappa} u^{\kappa}\left(u^{-\kappa} I_{t-}^{-\kappa} z^{\kappa} 1_{[0, s)}(z)\right)\right)(v)=1_{[0, s)}(v), B^{0}$ is the usual Brownian motion. By using the fractional integration by parts formula $\int_{0}^{t} \phi(s)\left(I_{t-}^{\alpha} \psi\right)(s) \mathrm{d} s=\int_{0}^{t}\left(I_{0+}^{\alpha} \phi\right)(s) \psi(s) \mathrm{d} s$ with $\left(I_{0+}^{\alpha} \phi\right)(s)=\Gamma(\alpha)^{-1} \int_{a}^{b} \phi(u)(s-u)_{+}^{\alpha-1} \mathrm{~d} u$, we can write $(7.1)$ as $B^{0}(s)=\int_{0}^{t} 1_{[0, s)}(z)$ $z^{\kappa}\left(I_{0+}^{-\kappa} u^{-\kappa} \dot{B}^{\kappa}(u)\right)(z) \mathrm{d} z$, where $\dot{B}^{\kappa}$ denotes the 'derivative' of $B^{\kappa}$, or

$$
\dot{B}^{0}(s)=s^{\kappa}\left(I_{0+}^{-\kappa} u^{-\kappa} \dot{B}^{\kappa}(u)\right)(s) .
$$

By applying the operator $u^{\kappa} I_{0+}^{\kappa} s^{-\kappa}$ to both sides of the last expression, integrating over $[0, s]$ and applying the fractional integration by parts formula again, we obtain

$$
B^{\kappa}(s)=\int_{0}^{t} u^{-\kappa}\left(I_{t-}^{\kappa} z^{\kappa} 1_{[0, s)}(z)\right)(u) \mathrm{d} B^{0}(u) .
$$

Since $B^{0}$ and $B^{\kappa}$ can be defined through each other as in (7.1) and (7.2), we obtain $\sigma\left\{B^{\kappa}(s), s \in[0, a]\right\}=\sigma\left\{B^{0}(s), s \in[0, a]\right\}$ up to sets of measure zero, where $\sigma\left\{B^{\kappa}(s)\right.$, $s \in[0, a]\}$ is the $\sigma$-algebra generated by the random variables $B^{\kappa}(s), s \in[0, a]$. Then, since $B^{0}$ is the usual Brownian motion,

$$
\begin{aligned}
\mathrm{E}\left(B^{\kappa}(t) \mid B^{\kappa}(s), s \in[0, a]\right) & =\mathrm{E}\left(\int_{0}^{t} u^{-\kappa}\left(I_{t-}^{\kappa} z^{\kappa} 1_{[0, t)}(z)\right)(u) \mathrm{d} B^{0}(u) \mid B^{0}(s), s \in[0, a]\right) \\
& =\int_{0}^{a} u^{-\kappa}\left(I_{t-}^{\kappa} z^{\kappa} 1_{[0, t)}(z)\right)(u) \mathrm{d} B^{0}(u) .
\end{aligned}
$$

By writing $1_{[0, t)}=1_{[0, a)}+1_{[a, t)}$ and then using (7.2), we obtain

$$
\mathrm{E}\left(B^{\kappa}(t) \mid B^{\kappa}(s), s \in[0, a]\right)=B^{\kappa}(a)+\int_{0}^{a} u^{-\kappa}\left(I_{t-}^{\kappa} z^{\kappa} 1_{[a, t)}(z)\right)(u) \mathrm{d} B^{0}(u) .
$$

We wish to rewrite this last integral as an integral with respect to FBM. Since, heuristically, $u^{-\kappa}\left(I_{t-}^{\kappa} z^{\kappa} 1_{[a, t)}(z)\right)(u)=u^{-\kappa} I_{a-}^{\kappa}\left(v^{\kappa} v^{-\kappa} I_{a-}^{-\kappa}\left(I_{t-}^{\kappa} z^{\kappa} 1_{[a, t)}(z)\right)(v)\right)(u)$ and since, for an integrable function $f, \int_{0}^{a} f(u) \mathrm{d} B^{\kappa}(u)=\int_{0}^{a} u^{-\kappa}\left(I_{a-}^{\kappa} z^{\kappa} f(z)\right)(u) \mathrm{d} B^{0}(u)$, we expect that 


$$
\mathrm{E}\left(B^{\kappa}(t) \mid B^{\kappa}(s), s \in[0, a]\right)=B^{\kappa}(a)+\int_{0}^{a} u^{-\kappa}\left(I_{a-}^{-\kappa}\left(I_{t-}^{\kappa} z^{\kappa} 1_{[a, t)}(z)\right)\right)(u) \mathrm{d} B^{\kappa}(u) .
$$

These computations suggest the following prediction formula for FBM:

Theorem 7.1. Let $0<a<t, \kappa \in\left(-\frac{1}{2}, \frac{1}{2}\right)$ and $B^{\kappa}$ be an FBM. Then

$$
\mathrm{E}\left(B^{\kappa}(t) \mid B^{\kappa}(s), s \in[0, a]\right)=B^{\kappa}(a)+\int_{0}^{a} \Psi_{t}(a, u) \mathrm{d} B^{\kappa}(u),
$$

where, for $u \in(0, a)$,

$$
\begin{aligned}
\Psi_{t}(a, u) & =u^{-\kappa}\left(I_{a-}^{-\kappa}\left(I_{t-}^{\kappa} z^{\kappa} 1_{[a, t)}(z)\right)\right)(u) \\
& =\frac{\sin \pi \kappa}{\pi} u^{-\kappa}(a-u)^{-\kappa} \int_{a}^{t} \frac{z^{\kappa}(z-a)^{\kappa}}{z-u} \mathrm{~d} z .
\end{aligned}
$$

Proof. Let us first verify that the right-hand sides of (7.4) and (7.5) are equal when $\kappa \in\left(0, \frac{1}{2}\right)$. Observe that, by $(2.1)$, for $v \in(0, a)$,

$$
\left(I_{t-}^{\kappa} z^{\kappa} 1_{[a, t)}(z)\right)(v)=\frac{1}{\Gamma(\kappa)} \int_{a}^{t} z^{\kappa}(z-v)^{\kappa-1} \mathrm{~d} z
$$

and hence, by (2.2),

$$
\begin{aligned}
\left(I_{a-}^{-\kappa}\left(I_{t-}^{\kappa} z^{\kappa} 1_{[a, t)}(z)\right)\right)(u) & =-\frac{1}{\Gamma(1-\kappa) \Gamma(\kappa)} \frac{\mathrm{d}}{\mathrm{d} u} \int_{u}^{a} \int_{a}^{t} z^{\kappa}(z-v)^{\kappa-1} \mathrm{~d} z(v-u)^{-\kappa} \mathrm{d} v \\
& =-\frac{\sin \pi \kappa}{\pi} \frac{\mathrm{d}}{\mathrm{d} u} \int_{a}^{t} \mathrm{~d} z z^{\kappa} \int_{u}^{a} \mathrm{~d} v(z-v)^{\kappa-1}(v-u)^{-\kappa}
\end{aligned}
$$

since $\Gamma(1-\kappa) \Gamma(\kappa)=\pi / \sin \pi \kappa$. By making the change of variables $v=u+(z-u) s$ and then taking $\mathrm{d} / \mathrm{d} u$ inside the integral, we obtain

$$
\begin{aligned}
\left(I_{a-}^{-\kappa}\left(I_{t-}^{\kappa} z^{\kappa} 1_{[a, t)}(z)\right)\right)(u) & =-\frac{\sin \pi \kappa}{\pi} \frac{\mathrm{d}}{\mathrm{d} u} \int_{a}^{t} \mathrm{~d} z z^{\kappa} \int_{0}^{(a-u) /(z-u)} \mathrm{d} s(1-s)^{\kappa-1} s^{-\kappa} \\
& =\frac{\sin \pi \kappa}{\pi}(a-u)^{-\kappa} \int_{a}^{t} \frac{z^{\kappa}(z-a)^{\kappa}}{z-u} \mathrm{~d} z .
\end{aligned}
$$

When $\kappa \in\left(-\frac{1}{2}, 0\right)$, the right-hand sides of (7.4) and (7.5) are still equal. To see this, observe first that (7.6) with $\Gamma(\kappa)=\Gamma(1+\kappa) / \kappa$ still holds for $v \in(0, a)$, since $z \neq v$ in the range of integration. Then (7.7) becomes (see (2.1))

$$
\begin{aligned}
\left(I_{a-}^{-\kappa}\left(I_{t-}^{\kappa} z^{\kappa} 1_{[a, t)}(z)\right)\right)(u) & =\frac{\kappa}{\Gamma(1+\kappa) \Gamma(-\kappa)} \int_{u}^{a} \int_{a}^{t} z^{\kappa}(z-v)^{\kappa-1} \mathrm{~d} z(v-u)^{-\kappa-1} \mathrm{~d} v \\
& =-\frac{\kappa \sin \pi \kappa}{\pi} \int_{a}^{t} \mathrm{~d} z z^{\kappa} \int_{u}^{a} \mathrm{~d} v(z-v)^{\kappa-1}(v-u)^{-\kappa-1} .
\end{aligned}
$$


It is now easy to see that

$$
\kappa \int_{u}^{a}(z-v)^{\kappa-1}(v-u)^{-\kappa-1} \mathrm{~d} v=\frac{\mathrm{d}}{\mathrm{d} u} \int_{u}^{a}(z-v)^{\kappa-1}(v-u)^{-\kappa} \mathrm{d} v=-\frac{(a-u)^{-\kappa}(z-a)^{\kappa}}{z-u},
$$

where the derivative can be computed as in the case $\kappa \in\left(0, \frac{1}{2}\right)$. This yields relation (7.8) in the case $\kappa \in\left(-\frac{1}{2}, 0\right)$ as well.

To verify that the integral in (7.3) is well defined for $\kappa \in\left(-\frac{1}{2}, 0\right)$, it is enough to check, by (1.8), that the function $s^{-\kappa}\left(I_{t-}^{\kappa} z^{\kappa} 1_{[a, t)}(z)\right)(s)$ belongs to $L^{2}[0, a]$. The same condition needs to be verified in the case $\kappa \in\left(0, \frac{1}{2}\right)$ by (1.2) and (2.5). In both cases this can be deduced from (7.6). Finally, to prove (7.3), it is enough to verify that, for all $s \in[0, a]$,

$$
\mathrm{E} B^{\kappa}(s)\left(B^{\kappa}(t)-B^{\kappa}(a)\right)=\mathrm{E} B^{\kappa}(s) \int_{0}^{a} \Psi_{t}(a, u) \mathrm{d} B^{\kappa}(u)
$$

This follows from (1.4) and (1.9) by using expression (7.4) for $\Psi_{t}(a, u)$.

\section{Proofs of Theorem 4.1 and Theorem 4.2}

We prove Theorem 4.1 and Theorem 4.2 together. Let $\kappa \in\left(-\frac{1}{2}, \frac{1}{2}\right)$. To show that the maps (1.4) and (1.9) (or (1.10)) define inner products on linear spaces $\Lambda_{a}^{\kappa}$, given by (1.2) and (1.8) respectively, we check the least obvious condition. If $(f, f)_{\Lambda_{a}^{k}}=0$ and $\kappa \in\left(0, \frac{1}{2}\right)$, then $\left(I_{a-}^{\kappa} u^{\kappa} f(u)\right)(s)=0$ a.e. $s \in[0, a]$. It follows by Lemma 2.5 in Samko et al. (1993, p. 40) that $u^{\kappa} f(u)=0$ a.e. $u \in[0, a]$ and hence that $f(u)=0$ a.e. $u \in[0, a]$. If $(f, f)_{\Lambda_{a}^{\kappa}}=0$ and $\kappa \in\left(-\frac{1}{2}, 0\right)$, then $\phi_{f}(s)=0$ a.e. $s \in[0, a]$, where $\phi_{f} \in L^{2}[0, a]$ is associated with the function $f$ by definition (1.8). It then follows that $f(u)=0$ a.e. $u \in[0, a]$ as well.

Let us show that the set of elementary functions $\mathcal{E}_{a}$ is dense in $\Lambda_{a}^{\kappa}$ when $\kappa \in\left(-\frac{1}{2}, \frac{1}{2}\right)$. Assume without loss of generality that $a>1$. Since any function $\phi \in L^{2}[0, a]$ can be approximated in $L^{2}[0, a]$ by functions $s^{-\kappa} \sum_{k=1}^{n} b_{k} 1_{\left[c_{k}, d_{k}\right)}(s)=s^{-\kappa} \sum_{k=1}^{n} b_{k}\left(1_{\left[0, d_{k}\right)}(s)-\right.$ $\left.1_{\left[0, c_{k}\right)}(s)\right)$ with $b_{k} \in \mathbb{R}$ and $0<c_{k}<d_{k}<a, k=1, \ldots, n$, it is enough to show, for example, that there is a sequence of elementary functions $f_{n} \in \mathcal{E}_{a} \subset \Lambda_{a}^{\kappa}$ such that

$$
h_{n}(\kappa):=\int_{0}^{a} s^{-2 \kappa}\left|1_{[0,1)}(s)-\left(I_{a-}^{\kappa} u^{\kappa} f_{n}(u)\right)(s)\right|^{2} \mathrm{~d} s \rightarrow 0, \quad n \rightarrow \infty .
$$

Since $1_{[0,1)}(s)=\left(I_{a-}^{\kappa} u^{\kappa} f(u)\right)(s)$ with $f(u)=u^{-\kappa}\left(I_{a-}^{-\kappa} 1_{[0,1)}\right)(u)=(\Gamma(1-\kappa))^{-1} u^{-\kappa}(1-u)_{+}^{-\kappa}$, the convergence (8.1) for some elementary functions $f_{n}$ follows from the following lemma.

Lemma 8.1. Let $\kappa \in\left(-\frac{1}{2}, \frac{1}{2}\right),\left\{B^{\kappa}(u)\right\}_{u \in[0,1]}$ be a standard FBM and

$$
f(u)=u^{-\kappa}\left(I_{1-}^{-\kappa} 1_{[0,1)}\right)(u)=(\Gamma(1-\kappa))^{-1} u^{-\kappa}(1-u)_{+}^{-\kappa} \text { for } u \in[0,1]
$$

Then the integral $\int_{0}^{1} f(u) \mathrm{d} B^{\kappa}(u)$ is the limit in the $L^{2}(\Omega)$ sense of the integrals

$$
\int_{0}^{1} f_{n}(u) \mathrm{d} B^{\kappa}(u)=\frac{1}{\Gamma(1-\kappa)} \sum_{l=2}^{n-2}\left(\frac{l}{n}\right)^{-\kappa}\left(1-\frac{l}{n}\right)^{-\kappa}\left(B^{\kappa}\left(\frac{l+1}{n}\right)-B^{\kappa}\left(\frac{l}{n}\right)\right),
$$


where, for $u \in[0,1]$,

$$
f_{n}(u)=\frac{1}{\Gamma(1-\kappa)} \sum_{l=2}^{n-2}\left(\frac{l}{n}\right)^{-\kappa}\left(1-\frac{l}{n}\right)^{-\kappa} 1_{\left[\frac{1}{n}, \frac{l+1}{n}\right)}(u)=\frac{1}{\Gamma(1-\kappa)} \sum_{l=2}^{n-2} f\left(\frac{l}{n}\right) 1_{\left[\frac{l}{n}, \frac{l+1}{n}\right)}(u) .
$$

Remark 8.1. The integral $\int_{0}^{1} f(u) \mathrm{d} B^{\kappa}(u)$ in Lemma 8.1 is of particular interest because, if $f_{t}(u)=u^{-\kappa}\left(I_{t-}^{-\kappa} 1_{[0, t)}\right)(u)=(\Gamma(1-\kappa))^{-1} u^{-\kappa}(t-u)_{+}^{-\kappa}, \quad t, u \in[0, a]$, then by (4.3) (and Theorems 4.1 and 4.2)

$$
\int_{0}^{t} f_{t}(u) \mathrm{d} B^{\kappa}(u) \stackrel{d}{=} \sigma_{1}(\kappa) \int_{0}^{t} s^{-2 \kappa} \mathrm{d} B^{0}(s), \quad t \in[0, a]
$$

The process on the left-hand side is known as the fundamental martingale (see Norros et al. 1999).

Proof. It is enough to prove the convergence (8.1) with $a=1$. If $\kappa \in\left(0, \frac{1}{2}\right)$, by using relation (4.5) and estimate (4.10), we obtain that

$$
h_{n}(\kappa) \leqslant \int_{0}^{1} s^{-2 \kappa}\left[\left(I_{1-}^{\kappa} u^{\kappa}\left|f(u)-f_{n}(u)\right|\right)(s)\right]^{2} \mathrm{~d} s \leqslant c(\kappa)\left\|f-f_{n}\right\|_{L^{2}[0,1]}^{2} \rightarrow 0,
$$

as $n \rightarrow \infty$.

The case $\kappa \in\left(-\frac{1}{2}, 0\right)$ is more delicate. We will show that $h_{n}(\kappa) \rightarrow 0$ as well, by applying the dominated convergence theorem. Let $l_{n}=l / n$ for $l, n \in \mathbb{N}$. As in (3.5) and (3.6), we obtain

$$
\begin{aligned}
\Gamma(1-\kappa) \Gamma(1+\kappa)\left(I_{1-}^{\kappa} u^{\kappa} f_{n}(u)\right)(s)= & \Gamma(1+\kappa) \sum_{l=2}^{n-2} l_{n}^{-\kappa}\left(1-l_{n}\right)^{-\kappa}\left(I_{1-}^{\kappa} u^{\kappa} 1_{\left[l_{n},(l+1)_{n}\right)}(u)\right)(s) \\
= & -\kappa \int_{s}^{1} \sum_{l=2}^{n-2} l_{n}^{-\kappa}\left(1-l_{n}\right)^{-\kappa} 1_{\left[l_{n},(l+1)_{n}\right)}(u) u^{\kappa-1}(u-s)_{+}^{\kappa} \mathrm{d} u \\
& +\sum_{l=2}^{n-2} l_{n}^{-\kappa}\left(1-l_{n}\right)^{-\kappa}\left((l+1)_{n}^{\kappa}\left((l+1)_{n}-s\right)_{+}^{\kappa}-l_{n}^{\kappa}\left(l_{n}-s\right)_{+}^{\kappa}\right) \\
= & : g_{n}^{1}(s)+g_{n}(s) .
\end{aligned}
$$

The summation by parts formula $\sum_{l=2}^{n-2} a_{l}\left(b_{l+1}-b_{l}\right)=-\sum_{l=2}^{n-2} b_{l}\left(a_{l}-a_{l-1}\right)+$ $a_{n-2} b_{n-1}-a_{1} b_{2}$ implies that 


$$
\begin{aligned}
g_{n}(s)= & -\sum_{l=2}^{n-2} l_{n}^{\kappa}\left(l_{n}-s\right)_{+}^{\kappa}\left(l_{n}^{-\kappa}\left(1-l_{n}\right)^{-\kappa}-(l-1)_{n}^{-\kappa}\left(1-(l-1)_{n}\right)^{-\kappa}\right) \\
& \left.+(n-2)_{n}^{-\kappa}\left(1-(n-2)_{n}\right)^{-\kappa}(n-1)_{n}^{\kappa}\left((n-1)_{n}-s\right)_{+}^{\kappa}-1_{n}^{-\kappa}(1-1)_{n}\right)^{-\kappa} 2_{n}^{\kappa}\left(2_{n}-s\right)_{+}^{\kappa} \\
= & -\sum_{l=2}^{n-2} l_{n}^{\kappa}\left(l_{n}-s\right)_{+}^{\kappa}\left(l_{n}^{-\kappa}-(l-1)_{n}^{-\kappa}\right)\left(1-l_{n}\right)^{-\kappa}-\sum_{l=2}^{n-2} l_{n}^{\kappa}\left(l_{n}-s\right)_{+}^{\kappa}(l-1)_{n}^{-\kappa}\left(\left(1-l_{n}\right)^{-\kappa}\right. \\
& \left.-\left(1-(l-1)_{n}\right)^{-\kappa}\right)+(n-2)_{n}^{-\kappa}\left(1-(n-2)_{n}\right)^{-\kappa}(n-1)_{n}^{\kappa}\left((n-1)_{n}-s\right)_{+}^{\kappa} \\
& -1_{n}^{-\kappa}\left(1-1_{n}\right)^{-\kappa} 2_{n}^{\kappa}\left(2_{n}-s\right)_{+}^{\kappa} \\
= & : g_{n}^{2}(s)+g_{n}^{3}(s)+g_{n}^{4}(s)+g_{n}^{5}(s) .
\end{aligned}
$$

(Recall that $l_{n}$ stands for $l / n$.) In order to establish (8.1), we will show that, for $\kappa \in\left(-\frac{1}{2}, 0\right)$,

$$
\begin{gathered}
\int_{0}^{1} s^{-2 \kappa}\left|g_{n}^{5}(s)\right|^{2} \mathrm{~d} s \rightarrow 0, \quad n \rightarrow \infty, \\
\Gamma(1-\kappa) \Gamma(1+\kappa)\left(I_{1-}^{\kappa} u^{\kappa} f_{n}(u)\right)(s)-g_{n}^{5}(s)=g_{n}^{1}(s)+g_{n}^{2}(s)+g_{n}^{3}(s)+g_{n}^{4}(s) \\
\rightarrow \Gamma(1-\kappa) \Gamma(1+\kappa)\left(I_{1-}^{\kappa} u^{\kappa} f(u)\right)(s)=\Gamma(1-\kappa) \Gamma(1+\kappa) 1_{[0,1)}(s) \text { a.e. } s \in[0,1],
\end{gathered}
$$

and

$$
\sup _{n}\left|g_{n}^{j}(s)\right| \leqslant g^{j}(s), \quad 1 \leqslant j \leqslant 4,
$$

where

$$
\int_{0}^{1} s^{-2 \kappa}\left|g^{j}(s)\right|^{2} \mathrm{~d} s<\infty, \quad 1 \leqslant j \leqslant 4 .
$$

Then, by the dominated convergence theorem, we will have the required convergence $h_{n}(\kappa) \rightarrow 0$ for $\kappa \in\left(-\frac{1}{2}, 0\right)$ as $n \rightarrow \infty$.

The convergence (8.2) follows, since

$$
\int_{0}^{1} s^{-2 \kappa}\left|g_{n}^{5}(s)\right|^{2} \mathrm{~d} s \leqslant c \int_{0}^{2 / n} s^{-2 \kappa}\left(\frac{2}{n}-s\right)^{2 \kappa} \mathrm{d} s=c \frac{2}{n} \int_{0}^{1} z^{-2 \kappa}(1-z)^{2 \kappa} \mathrm{d} z \rightarrow 0,
$$

as $n \rightarrow \infty$. Let us show (8.4) and (8.5) with $j=1$. We have

$$
\begin{aligned}
\left|g_{n}^{1}(s)\right| & \leqslant c_{1} \int_{s}^{1} \sum_{l=2}^{n-2} l_{n}^{-\kappa}\left(1-(l+1)_{n}\right)^{-\kappa} 1_{\left[l_{n},(l+1)_{n}\right)}(u) u^{\kappa-1}(u-s)_{+}^{\kappa} \mathrm{d} u \\
& \leqslant c_{1} \int_{s}^{1} u^{-\kappa}(1-u)^{-\kappa} u^{\kappa-1}(u-s)_{+}^{\kappa} \mathrm{d} u=c_{1} \int_{s}^{1} u^{-1}(1-u)^{-\kappa}(u-s)_{+}^{\kappa} \mathrm{d} u=: g^{1}(s),
\end{aligned}
$$

where the constant $c_{1}$ depends on $\kappa$ only. It is enough to check the convergence of the 
integral in (8.5) around $s=0$ only. Since $\kappa<0$, observe that, as $s$ is close to 0 , the function $g^{1}(s)$ is bounded (up to a constant) by

$$
\int_{s}^{1} u^{-1}(u-s)^{\kappa} \mathrm{d} u=s^{\kappa} \int_{1}^{1 / s} v^{-1}(v-1)^{\kappa} \mathrm{d} v \leqslant c s^{\kappa} \int_{1}^{\infty} v^{\kappa-1} \mathrm{~d} v \leqslant \frac{c}{|\kappa|} s^{\kappa} .
$$

It then follows that the integral in (8.5) with $j=1$ converges around $s=0$. For functions $g_{n}^{2}$, by using the inequality $\left|l_{n}^{-\kappa}-(l-1)_{n}^{-\kappa}\right| \leqslant|\kappa|(l-1)_{n}^{-\kappa-1} / n$, we have

$$
\begin{aligned}
\left|g_{n}^{2}(s)\right| & \leqslant|\kappa| \sum_{l=2}^{n-2} l_{n}^{\kappa}\left(l_{n}-s\right)_{+}^{\kappa}(l-1)_{n}^{-\kappa-1}\left(1-l_{n}\right)^{-\kappa} \frac{1}{n} \\
& \leqslant c_{2} \sum_{l=2}^{n-2}(l+1)_{n}^{-1}\left(l_{n}-s\right)_{+}^{\kappa}\left(1-(l+1)_{n}\right)^{-\kappa} \frac{1}{n} \\
& \leqslant c_{2} \int_{0}^{1} u^{-1}(u-s)_{+}^{\kappa}(1-u)^{-\kappa} \mathrm{d} u=: g^{2}(s),
\end{aligned}
$$

where the constant $c_{2}$ depends on $\kappa$ only. Relation (8.5) with $j=2$ follows as in the case $j=1$ since the functions $g_{1}$ and $g_{2}$ are equal up to a constant. As for the functions $g_{n}^{3}$, one can similarly show that

$$
\left|g_{n}^{3}(s)\right| \leqslant c_{3} \int_{0}^{1}(u-s)_{+}^{\kappa}(1-u)^{-\kappa-1} \mathrm{~d} u=c_{3} \int_{0}^{1} z^{\kappa}(1-z)^{-\kappa-1} \mathrm{~d} u=c_{3} \mathrm{~B}(1+\kappa,-\kappa)=: g^{3}(s)
$$

and

$$
\int_{0}^{1} s^{-2 \kappa}\left|g^{3}(s)\right|^{2} \mathrm{~d} s=c_{3}^{2} \mathrm{~B}(1+\kappa,-\kappa)^{2} \int_{0}^{1} s^{-2 \kappa} \mathrm{d} s<\infty
$$

The proof of (8.4) and (8.5) with $j=4$ is obvious. Finally, to show the convergence (8.3), observe that

$$
\begin{aligned}
\lim _{n \rightarrow \infty}( & \left.(1-\kappa) \Gamma(1+\kappa)\left(I_{1-}^{\kappa} u^{\kappa} f_{n}(u)\right)(s)-g_{n}^{5}(s)\right) \\
= & \lim _{n \rightarrow \infty}\left(g_{n}^{1}(s)+g_{n}^{2}(s)+g_{n}^{3}(s)+g_{n}^{4}(s)\right) \\
= & -\kappa \int_{0}^{1} u^{-\kappa}(u-s)_{+}^{\kappa} u^{\kappa-1}(1-u)^{-\kappa} \mathrm{d} u+\kappa \int_{0}^{1} u^{\kappa}(u-s)_{+}^{\kappa} u^{-\kappa-1}(1-u)^{-\kappa} \mathrm{d} u \\
& -\kappa \int_{0}^{1} u^{\kappa}(u-s)_{+}^{\kappa} u^{-\kappa}(1-u)^{-\kappa-1} \mathrm{~d} u+0=-\kappa \int_{0}^{1}(u-s)_{+}^{\kappa}(1-u)^{-\kappa-1} \mathrm{~d} u \\
= & -\kappa \mathrm{B}(\kappa+1,-\kappa) 1_{[0,1)}(s)=\Gamma(1-\kappa) \Gamma(1+\kappa) 1_{[0,1)}(s), \text { a.e. } s \in[0,1] .
\end{aligned}
$$




\section{Appendix}

In the proof of Proposition 3.1, we used the fact that the function

$$
f(\kappa)=\sigma_{1}(\kappa)^{2} \int_{0}^{a} s^{-2 \kappa}\left(I_{a-}^{\kappa} u^{\kappa} 1_{\left[0, t_{1}\right)}(u)\right)(s)\left(I_{a-}^{\kappa} u^{\kappa} 1_{\left[0, t_{2} 0\right.}(u)\right)(s) \mathrm{d} s,
$$

where $t_{1}, t_{2} \in[0, a]$, is analytic for complex $|\kappa|<\frac{1}{2}$. The analyticity of $f$ can be verified directly as follows. By (3.6), the function $f$ equals

$$
\begin{aligned}
f(\kappa)= & (\Gamma(1+\kappa))^{-2} \sigma_{1}(\kappa)^{2} \int_{0}^{a} s^{-2 \kappa}\left\{\kappa \int_{s}^{t_{1}} u^{\kappa-1}(u-s)_{+}^{\kappa} \mathrm{d} u-t_{1}^{\kappa}\left(t_{1}-s\right)_{+}^{\kappa}\right\} \\
& \times\left\{\kappa \int_{s}^{t_{2}} u^{\kappa-1}(u-s)_{+}^{\kappa} \mathrm{d} u-t_{2}^{\kappa}\left(t_{2}-s\right)_{+}^{\kappa}\right\} \mathrm{d} s,
\end{aligned}
$$

where $\sigma_{1}(\kappa)^{2}$ is defined by (3.2). Since $(\Gamma(1+\kappa))^{-2} \sigma_{1}(\kappa)^{2}$ is analytic on $|\kappa|<\frac{1}{2}$, it is enough to show that the function

$$
\begin{aligned}
\int_{0}^{a} s^{-2 \kappa}\left\{\kappa \int_{s}^{t_{1}} u^{\kappa-1}(u-s)_{+}^{\kappa} \mathrm{d} u-t_{1}^{\kappa}\left(t_{1}-s\right)_{+}^{\kappa}\right\}\left\{\kappa \int_{s}^{t_{2}} u^{\kappa-1}(u-s)_{+}^{\kappa} \mathrm{d} u-t_{2}^{\kappa}\left(t_{2}-s\right)_{+}^{\kappa}\right\} \mathrm{d} s \\
=\kappa^{2} \int_{0}^{a} s^{-2 \kappa} \int_{s}^{t_{1}} u^{\kappa-1}(u-s)_{+}^{\kappa} \mathrm{d} u \int_{s}^{t_{2}} u^{\kappa-1}(u-s)_{+}^{\kappa} \mathrm{d} u \mathrm{~d} s-\kappa \int_{0}^{a} s^{-2 \kappa} \int_{s}^{t_{1}} u^{\kappa-1}(u-s)_{+}^{\kappa} \mathrm{d} u t_{2}^{\kappa}\left(t_{2}-s\right)_{+}^{\kappa} \mathrm{d} s \\
\quad-\kappa \int_{0}^{a} s^{-2 \kappa} \int_{s}^{t_{2}} u^{\kappa-1}(u-s)_{+}^{\kappa} \mathrm{d} u t_{1}^{\kappa}\left(t_{1}-s\right)_{+}^{\kappa} \mathrm{d} s+\kappa \int_{0}^{a} s^{-2 \kappa} t_{1}^{\kappa}\left(t_{1}-s\right)_{+}^{\kappa} t_{2}^{\kappa}\left(t_{2}-s\right)_{+}^{\kappa} \mathrm{d} s \\
=: \kappa^{2} f_{1}(\kappa)-\kappa f_{2}(\kappa)-\kappa f_{3}(\kappa)+f_{4}(\kappa)
\end{aligned}
$$

is analytic on $|\kappa|<\frac{1}{2}$, or, equivalently, that the functions $f_{1}(\kappa), f_{2}(\kappa), f_{3}(\kappa)$ and $f_{4}(\kappa)$ are analytic on $|\kappa|<\frac{1}{2}$. Assume without loss of generality that $t_{1}<t_{2}$ and consider the function $f_{4}(\kappa)=\int_{0}^{t_{1}} \mathrm{e}^{\kappa \ln s^{-2} t_{1}\left(t_{1}-s\right) t_{2}\left(t_{2}-s\right)} \mathrm{d} s$ first. For $h \in \mathbb{C}, h \neq 0$, we have that

$$
\frac{1}{h}\left(f_{4}(\kappa+h)-f_{4}(\kappa)\right)=\int_{0}^{t_{1}} f_{4}(\kappa, h, s) \mathrm{d} s,
$$

where

$$
f_{4}(\kappa, h, s)=\mathrm{e}^{\kappa \ln s^{-2} t_{1}\left(t_{1}-s\right) t_{2}\left(t_{2}-s\right)} \frac{\mathrm{e}^{h \ln s^{-2} t_{1}\left(t_{1}-s\right) t_{2}\left(t_{2}-s\right)}-1}{h} .
$$

Observe now that, for $u>0$ and $h=h_{1}+\mathrm{i} h_{2}$,

$$
\frac{\mathrm{e}^{\mathrm{i} h \ln u}-1}{h}=\frac{\mathrm{e}^{h_{1} \ln u} \mathrm{e}^{\mathrm{i} h_{2} \ln u}-1}{h_{1}+\mathrm{i} h_{2}}=\mathrm{e}^{\mathrm{i} h_{2} \ln u} \frac{\mathrm{e}^{h_{1} \ln u}-1}{h_{1}+\mathrm{i} h_{2}}+\frac{\mathrm{e}^{\mathrm{i} h_{2} \ln u}-1}{h_{1}+\mathrm{i} h_{2}}
$$

and hence that $|h|^{-1}\left|\mathrm{e}^{h \ln u}-1\right| \leqslant 2|\ln u| \mathrm{e}^{\left|h_{1} \ln u\right|} \leqslant 2|\ln u| \mathrm{e}^{\epsilon|\ln u|}$, if $|h|<\epsilon$. This implies that

$$
|h|^{-1}\left|\mathrm{e}^{h \ln s^{-2} t_{1}\left(t_{1}-s\right) t_{2}\left(t_{2}-s\right)}-1\right| \leqslant 2\left|\ln s^{-2} t_{1}\left(t_{1}-s\right) t_{2}\left(t_{2}-s\right)\right| \mathrm{e}^{\epsilon\left|\ln s^{-2} t_{1}\left(t_{1}-s\right) t_{2}\left(t_{2}-s\right)\right|},
$$

for $|h|<\epsilon$. Choose $\epsilon>0$ so that $\int_{0}^{1} s^{-2 \kappa} s^{-2 \epsilon} \mathrm{d} s<\infty$. Since 


$$
\int_{0}^{t_{1}} s^{-2 \kappa_{1}} t_{1}^{\kappa_{1}}\left(t_{1}-s\right)_{+}^{\kappa_{1}} t_{2}^{\kappa_{1}}\left(t_{2}-s\right)_{+}^{\kappa_{1}}\left|\ln s^{-2} t_{1}\left(t_{1}-s\right) t_{2}\left(t_{2}-s\right)\right| \mathrm{e}^{\epsilon\left|\ln s^{-2} t_{1}\left(t_{1}-s\right) t_{2}\left(t_{2}-s\right)\right|} \mathrm{d} s<\infty,
$$

where $\kappa_{1}$ is the real part of $\kappa$ and $|\kappa|<\frac{1}{2}$, we obtain from the dominated convergence theorem that

$\frac{\mathrm{d}}{\mathrm{d} \kappa} f_{4}(\kappa)=\lim _{h \rightarrow 0} \frac{f_{4}(\kappa+h)-f_{4}(\kappa)}{h}=\int_{0}^{t_{1}} s^{-2 \kappa} t_{1}^{\kappa}\left(t_{1}-s\right)_{+}^{\kappa} t_{2}^{\kappa}\left(t_{2}-s\right)_{+}^{\kappa} \ln s^{-2} t_{1}\left(t_{1}-s\right) t_{2}\left(t_{2}-s\right) \mathrm{d} s$ and hence that the function $f_{4}$ is analytic on $|\kappa|<\frac{1}{2}$.

For the function $f_{3}(\kappa)=\int_{0}^{t_{1}} \mathrm{e}^{\kappa \ln s^{-2} t_{1}\left(t_{1}-s\right)} \int_{s}^{t_{2}} u^{-1} \mathrm{e}^{\kappa \ln u(u-s)} \mathrm{d} u \mathrm{~d} s$, write

$$
\begin{aligned}
\frac{f_{3}(\kappa+h)-f_{3}(\kappa)}{h}= & \int_{0}^{t_{1}} \mathrm{e}^{\kappa \ln s^{-2} t_{1}\left(t_{1}-s\right)} \frac{\mathrm{e}^{h \ln s^{-2} t_{1}\left(t_{1}-s\right)}-1}{h} \int_{s}^{t_{2}} u^{-1} \mathrm{e}^{(\kappa+h) \ln u(u-s)} \mathrm{d} u \mathrm{~d} s \\
& +\int_{0}^{t_{1}} \mathrm{e}^{\kappa \ln s^{-2} t_{1}\left(t_{1}-s\right)} \int_{s}^{t_{2}} u^{-1} \mathrm{e}^{\kappa \ln u(u-s)} \frac{\mathrm{e}^{h \ln u(u-s)}-1}{h} \mathrm{~d} u \mathrm{~d} s
\end{aligned}
$$

and use the arguments above to conclude that

$$
\begin{aligned}
\frac{\mathrm{d}}{\mathrm{d} \kappa} f_{3}(\kappa)= & \int_{0}^{t_{1}} s^{-2 \kappa} t_{1}^{\kappa}\left(t_{1}-s\right)^{\kappa} \ln s^{-2} t_{1}\left(t_{1}-s\right) \int_{s}^{t_{2}} u^{\kappa-1}(u-s)^{\kappa} \mathrm{d} u \mathrm{~d} s \\
& +\int_{0}^{t_{1}} s^{-2 \kappa} t_{1}^{\kappa}\left(t_{1}-s\right)^{\kappa} \int_{s}^{t_{2}} u^{\kappa-1}(u-s)^{\kappa} \ln u(u-s) \mathrm{d} u \mathrm{~d} s
\end{aligned}
$$

and hence that the function $f_{3}$ is analytic on $|\kappa|<\frac{1}{2}$. The proof that the functions $f_{2}$ and $f_{1}$ are analytic on $|\kappa|<\frac{1}{2}$ is similar.

\section{Acknowledgement}

This research was partially supported by NSF grant ANI-9805623 at Boston University.

\section{References}

Carmona, P., Coutin, L. and Montseny, G. (1999) Stochastic integration with respect to fractional Brownian motion. Preprint.

Conway, J.B. (1978) Functions of One Complex Variable I. New York: Springer-Verlag.

Decreusefond, L. and Üstünel, A.S. (1999) Stochastic analysis of the fractional Brownian motion. Potential Anal., 10, 177-214.

Duncan, T.E., Hu, Y. and Pasik-Duncan, B. (2000) Stochastic calculus for fractional Brownian motion I: Theory. SIAM J. Control Optim., 38(2), 562-612.

Gripenberg, G. and Norros, I. (1996) On the prediction of fractional Brownian motion. J. Appl. Probab., 33, 400-410.

Kleptsyna, M.L., LeBreton, A. and Roubaud, M.C. (1999a) An elementary approach to filtering in systems with fractional Brownian observation noise. In B. Grigelionis, J. Kubilius, V. Paulauskas, 
H. Pragarauskas and V. Statulevicius (eds), Probability Theory and Mathematical Statistics: Proceedings of the 7th Vilnius Conference. Zeist: VSP.

Kleptsyna, M.L., LeBreton, A. and Roubaud, M.C. (1999b) Rudiments of stochastic fractional calculus and statistical applications. Preprint.

Norros, I., Valkeila, E. and Virtamo, J. (1999) An elementary approach to a Girsanov formula and other analytical results on fractional Brownian motions. Bernoulli, 5, 571-587.

Pipiras, V. and Taqqu, M.S. (2000) Integration questions related to fractional Brownian motion. Probab. Theory Related Fields, 118(2), 251-291.

Samko, S.G., Kilbas, A.A. and Marichev, O.I. (1993) Fractional Integrals and Derivatives: Theory and Applications. New York: Gordon and Breach.

Samorodnitsky, G. \& Taqqu, M.S. (1994) Stable Non-Gaussian Processes: Stochastic Models with Infinite Variance. London: Chapman \& Hall.

Received April 2000 and revised March 2001 